NASA TECHNICAL

MEMORANDUM

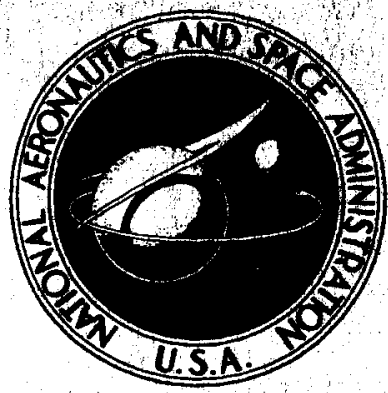

NASA TM X-1353
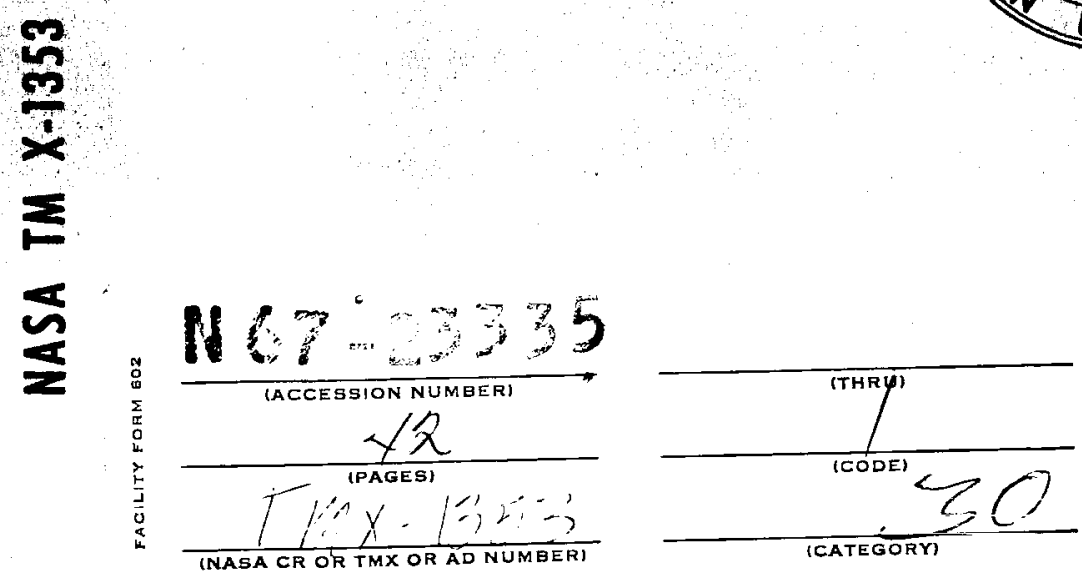

NECESSARY CONDITIONS FOR

OPTIMAL LUNAR TRAJECTORIES WITH

DISCONTINUOUSS STATE VARIABLES AND

INTERMEDIATE POINT CONSTRAINTS

by

W. E. Miner

Electronics Research Center

and

J. F. Andrus

Northrop Space Laboratories

National aeronautics and SPACE adMINISTRATION - WASHINGTON, D. C. • APRIL 1967 
By W. E. Miner

\author{
Electronics Research Center \\ Cambridge, Mass. \\ and \\ J. F. Andrus \\ Northrop Space Laboratories \\ Huntsville, Ala.
}




\title{
NECESSARY CONDITIONS FOR OPTIMAL LUNAR TRAJECTORIES WITH DISCONTINUOUS STATE VARIABLES AND INTERMEDIATE POINT CONSTRAINTS
}

\author{
by \\ W. E. Miner* and J. F. Andrus ${ }^{+}$
}

\section{SUMMARY}

The guidance regime for an optimal multi-stage lunar trajectory is derived by applying the mathematics of the calculus of variations as established by Denbow for the generalized problem of Bolza. The steering angle programs for four constant thrust level phases and the times to initiate and terminate the two coast phases are determined in order to place maximum payload into a specified lunar orbit. The problem considered here is to determine an optimal trajectory consisting of six sub-arcs utilizing three vehicle stages on which maximum payload is transported from an exo-atmospheric point near the Earth to a specified lunar orbit. The intermediate point constraints include two points at which stages are separated and mass discontinuities occur, an Earth parking orbit of specified energy and angular momentum magnitude, and four thrust magnitude levels. The Euler-Lagrange equations determine the optimal steering for the thrusting phases and the Denbow transversality equations are used to calculate the discontinuities at the ends of the subarcs. This method is applied here and the equations necessary to solve this problem using a high-speed computer are derived.

* Chief, Guidance Theory and Trajectory Analysis Branch, Electronics Research Center, NASA, Cambridge, Mass. Formerly with the Aero-Astrodynamics Laboratory, Marshall Space Flight Center, NASA, where the work represented in this paper was initiated.

+ Northrop Space Laboratories, Huntsville, Ala. Work performed while with the Huntsville Operation of the General Electric Computer Department under contract to the Computation Laboratory, Marshall Space Flight Center, NASA. 


\section{INTRODUCTION}

The calculus of variations is a well established mathematical tool for the determination of control functions and control parameters for optimal trajectories. The Euler-Lagrange equations yield the necessary conditions for extremal solutions to the split boundary value problem of Bolza. Extensions to the theory to provide for intermediate point constraints, such as those introduced into the system by the separation of vehicle stages or the initiation of a coast phase, were made by Denbow.

In general, the path of an aerospace vehicle can be described by a set of n nonlinear differential equations

$$
\dot{x}_{i}=E_{i}(\bar{x}(t), \bar{\alpha}(t), \bar{a}) \quad i=1,2, \ldots, n
$$

where

$\overline{\mathbf{x}}(\mathrm{t})$ is an $\mathrm{n}$ vector of state variables,

$$
\left(x_{1}, x_{2}, \ldots, x_{n}\right)^{T}
$$

$\bar{\alpha}(t)$ is an $m$ vector of control functions,

$$
\left(\alpha_{1}, \alpha_{2}, \ldots, \alpha_{m}\right)^{\mathrm{T}}
$$

$\bar{a}$ is a $k$ vector of control parameters,

$$
\left(a_{1}, a_{2}, \ldots, a_{k}\right)^{T}
$$

and $T$ means transposed. A particular path results when the initial conditions $\bar{x}\left(t_{0}\right)$, the control functions $\bar{\alpha}(t)$ for $t o t \leq t_{f}$, and the control numbers, $\bar{a}$, are given where $t_{o}$ and $t_{f}$ are the initial and final times.

An optimal trajectory is one which results when $\bar{\alpha}(t)$ and $\bar{a}$ are chosen so that a specified terminal quantity $\phi\left(x\left(t_{f}\right), t_{f}\right)$, called the pay-off, is maximized or minimized and a specified $p$ vector of terminal constraints

$$
\psi_{j}\left(x\left(t_{f}\right), t_{f}\right)=0 \quad j=1,2, \ldots, p
$$

is satisfied. 
The calculus of variations provides for the solution of such problems by introducing the $n$ vector function $\lambda(t)$ of Lagrange multipliers, which satisfies the set of linear differential equations

$$
\dot{\lambda}_{i}=-\sum_{j=1}^{n} \frac{\partial f_{j}}{\partial x_{i}} \lambda_{j} \quad i=1,2, \ldots, n
$$

subject to the $\mathrm{m}$ relations

$$
\sum_{j=1}^{n} \lambda_{j} \frac{\partial f_{j}}{\partial \alpha_{k}}=0 \quad k=1,2, \ldots, m
$$

as well as appropriate terminal conditions.

The problem is complicated by the presence of intermediate point constraints. These divide the trajectory into a sequence of sub-arcs. Specifying the values of state variables or control functions at intermediate points imposes constraints on the Lagrange multipliers at these points. The calculation of these constraints at the ends of the sub-arcs so that the composite trajectory is an extremal was made by Denbow. ${ }^{1}$ His results can be applied to intermediate points at which state variables or control functions are subject to specified finite discontinuities, e.g., the cases of vehicle staging and switching from powered flight to coast. These necessary conditions at the ends of the sub-arcs, which are called transversality conditions, can be written in an elegant form as upper bounds on the rank of a prescribed matrix.

This paper is an application of the theory of optimal control with intermediate point constraints to a specific lunar trajectory problem. This multistage trajectory is reduced to a two-point boundary value problem and the results comprise the necessary conditions for an extremum. In order to obtain a specific optimal lunar trajectory, it is now necessary to solve the two-point boundary value problem. Numerical methods for converging to a solution, i.e., an extremal trajectory, have been described widely in the literature.

1 Denbow, C. H. : A Generalized Form of the Problem of Bolza. Contributions to the Calculus of Variations, University of Chicago Press, Oct. 1937. 
A list of symbols used in this Technical Memorandum is presented below:

List of Symbols

A Augmented matrix shown in Figure 3

$\left.\begin{array}{l}A^{*} \\ A^{* *} \\ A^{* * *}\end{array}\right\}$

$\mathrm{C}$

Sub-matrices of A defined on pp. 24, 25, and 27

Matrix which relates Earth-centered and moon-centered coordinate systems

$\mathrm{C}_{3} \quad$ Sub-matrix of $\mathrm{A}$ associated with the parking-orbit constraints defined on p. 23

$\mathrm{C}_{3}{ }^{*} \quad$ Non-singular submatrix of $\mathrm{C}_{3}$

$\mathrm{C}_{7} \quad$ Sub-matrix of $\mathrm{A}$ associated with the lunar orbit constraints

$\mathrm{C}_{7}{ }^{*} \quad$ Non-singular sub-matrix of $\mathrm{C}_{7}$

$\mathrm{E}_{1}$

$\left.\begin{array}{l}\mathrm{E}_{2} \\ \mathrm{E}_{3}\end{array}\right\}$

E* $\quad$ Matrix defined on p. 35

$F_{i} \quad$ Vehicle thrust magnitude during $i^{\text {th }}$ flight phase

$\mathrm{i}=1,2, \ldots, 6$

$\overline{\mathrm{G}} \quad$ Gravitational force

$\mathrm{H}_{\mathrm{i}} \quad$ The Hamiltonian evaluated at point $\mathrm{i}$

$\mathrm{H}^{*} \quad$ Expression defined on $\mathrm{p} .33$

I Identity matrix

$I_{\text {sp }} \quad$ Specific impulse

M Sub-matrix of A defined on p. 28

M* Product matrix defined on p. 33

O Matrix of zeros, dimensions indicated

R Distance to entry point of parking orbit

S Euler-Lagrange sum defined on p. 15 
Flight duration from exit of Earth parking orbit to entrance of lunar orbit or matrix transpose when used as a superscript

Speed at entry point of parking orbit

$\left.\begin{array}{l}X \\ Y \\ z\end{array}\right\}$

a

$a_{i j}$

$\mathrm{c}_{\mathbf{i}}$

e

$\mathbf{f}_{\mathbf{i}}$

$\mathrm{g}_{\mathrm{o}}$

$\ell$

$\mathrm{m}_{\mathrm{i}}$

$\mathrm{r}_{\mathrm{s}}$

$\mathbf{s}_{\mathbf{j}}$

$t_{i}$

$\left.\begin{array}{l}u \\ v \\ w\end{array}\right\}$

$\bar{u}_{\mathrm{i}}$

$\overline{\mathrm{u}}_{\mathrm{s}}$

$\left.\begin{array}{l}x \\ y \\ z\end{array}\right\}$

$x(t)$

$\bar{x}_{\mathbf{i}}$

$\bar{x}_{s}$

$\bar{x}$

Cartesian coordinate components of $\bar{G}$

$\mathrm{k}$ vector of control parameters

General element of A

Quantities constrained at sub-arc boundaries

Eccentricity of lunar orbit

$\mathrm{n}$ vector of rates of state variables

Gravitational constant at surface of Earth

Semi-latus rectum of lunar orbit

Variable vehicle mass at point $\mathbf{i}$

Magnitude of position vector in moon-centered coordinate system

Time and state variables at sub-arc boundaries

Time at point $\mathrm{i}$

Vehicle velocity components in Cartesian coordinate system

Vehicle velocity vector at point i

Vehicle velocity vector in moon-centered coordinate system

Vehicle position compone nts in Cartesian coordinate system

General $\mathbf{n}$ vector of state variables

Vehicle position vector at point $i$

Vehicle position vector in moon-centered coordinate system

Position of moon with respect to Earth-centered reference system at point 7 
$\Lambda \quad$ Quantity defined on p. 17

$\alpha(t) \quad m$ vector of control functions

$\beta_{i} \quad$ Fuel burning rate during the $i^{\text {th }}$ flight phase

$\left.\delta_{p}\right\} \quad \pm 1$, used to indicate sign ambiguity

$\left.\delta_{y}^{p}\right\}$

$\lambda_{i}$

$\bar{\lambda}$

The ith $^{\text {th }}$ Lagrange multiplier

The vector $\left(\lambda_{1}, \lambda_{2}, \lambda_{3}\right)^{\mathrm{T}}$ evaluated at point $\mathrm{i}$

He Gravitational constant of moon

$\bar{\rho}_{i}$

$\bar{\rho}$ *

The vector $\left(\lambda_{4}, \lambda_{5}, \lambda_{6}\right)^{\mathrm{T}}$ evaluated at point i

Expression defined on p. 33

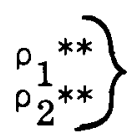

$\tau$

$\phi$

$x_{\rho}$

$x_{y}$

$\psi_{j}$

$\psi_{i}$

$\bar{\psi}$

Expressions defined on p. 36

General variable used to represent, in turn, each of the state variables and control functions

Terminal quantity to be maximized or minimized, the payoff

Thrust angle in pitch control variables

Thrust angle in yaw $\}$ shown in Figure 2

General terminal constraint

Constraint describing specified lunar orbit

Vector $\left[\psi_{1}, \psi_{2}, \ldots, \psi_{5}\right]^{\mathrm{T}}$. 


\section{THE PHYSICAL PROBLEM}

The vehicle is a variable mass particle which moves from a point outside the Earth's atmosphere into a specified lunar orbit under the action of its own thrust and a gravitational field computed using an ephemeris. The problem is to program the thrust steering for the powered phases and the times of initiation and termination of the coast phases so that the payload placed in orbit about the moon is maximum.

A non-rotating, Earth-centered Cartesian coordinate system is the principal frame of reference. The terminal orbit is given in a moon-centered system which is described when it is introduced.

Conditions are imposed at seven points along the trajectory as shown in Figure 1. These divide the flight path into six sub-arcs on each of which the magnitude of the thrust is constant and designated $\mathrm{F}_{1}, \mathrm{~F}_{2}, \mathrm{~F}_{4}$, and $\mathrm{F}_{6}$ on the powered arcs. The two coast periods correspond to $\mathrm{F}_{3}=\mathrm{F}_{5}=0$. The mass rate is a constant on each subarc. The vehicle has three stages so that the mass has a specified discontinuity at two points equal to the mass of the separated stage. Further, the third sub-arc is a parking orbit about the Earth which is only partially specified so that the remaining quantities may also be optimized.

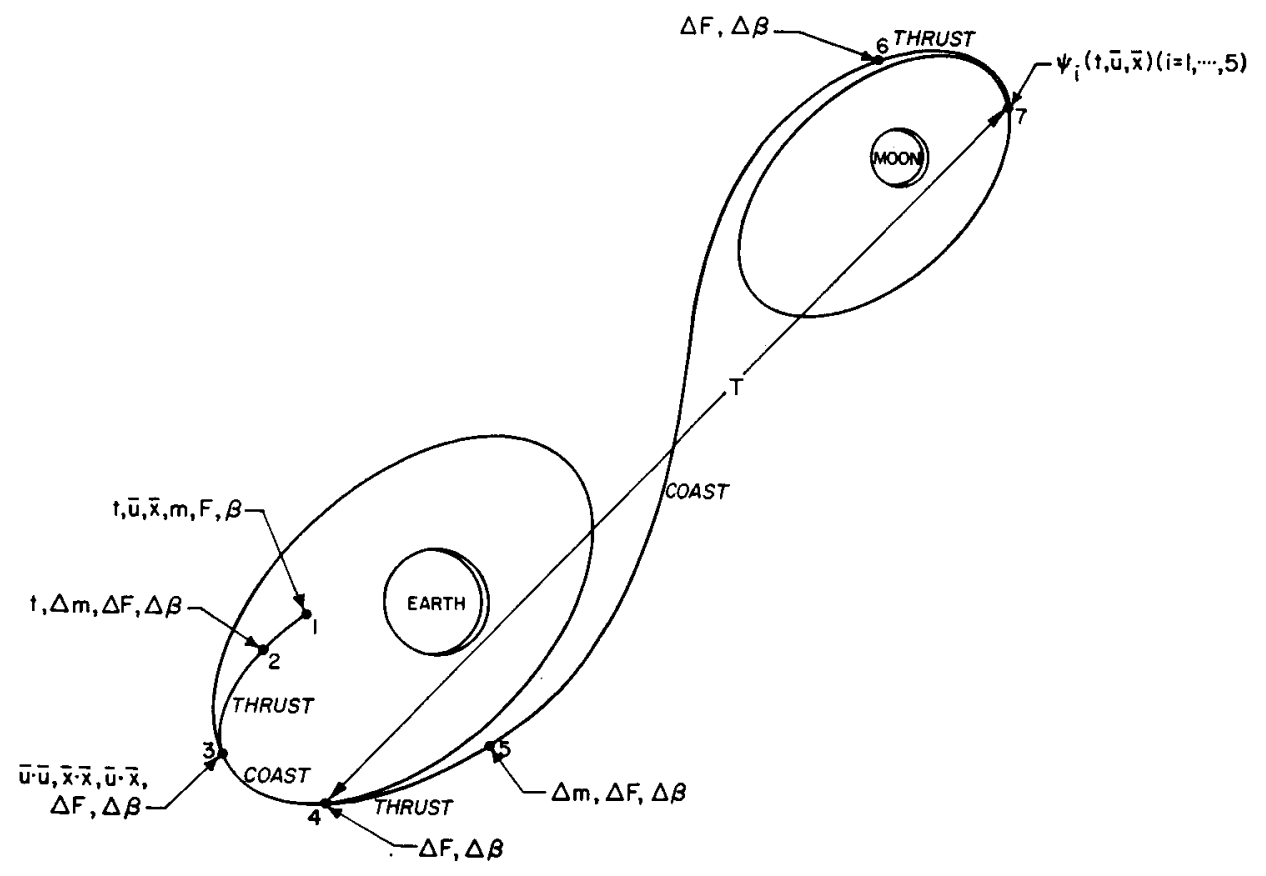

Figure 1. - Lunar trajectory for three-stage vehicle with powered and coasting arcs 
The equations of motion are:

$$
\begin{aligned}
& \dot{u}=-\frac{F}{m} \sin x_{p} \cos x_{y}+x \\
& \dot{v}=\frac{F}{m} \cos x_{p} \cos x_{y}+Y \\
& \dot{w}=\frac{F}{m} \sin x_{y}+z \\
& \dot{x}=u \\
& \dot{y}=v \\
& \dot{z}=w \\
& \dot{m}=-\beta
\end{aligned}
$$

where $\bar{x}=(x, y, z)^{T}$ is the position vector, $\bar{u}=(u, v, w)^{T}$ is the velocity vector, $F$ and $\beta$ are specified constants different on each sub-arc, $m$ is the mass, $x_{p}$ and $x_{y}$ are the steering angles for the thrust vector, as shown in Figure 2, and $X, Y$, $Z$ are the components of the gravitational field. We seek $x_{p}(t)$ and $x_{y}(t)$ and the times to initiate and terminate the coast phases.

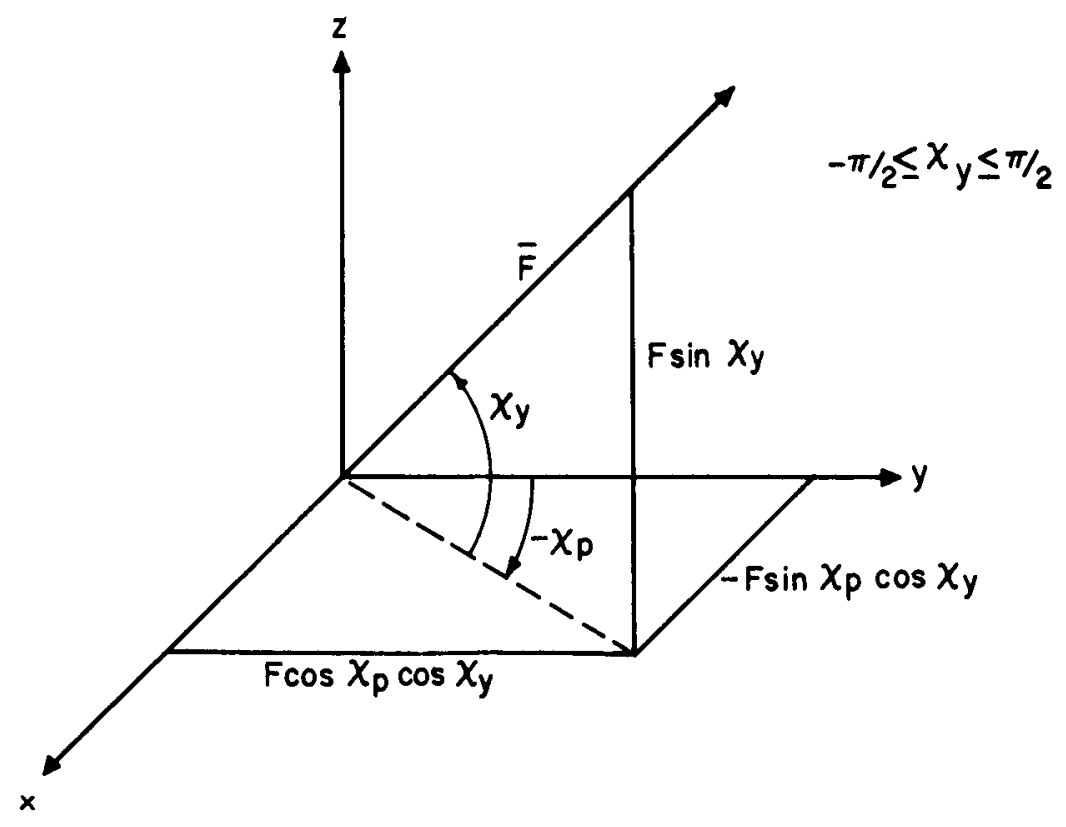

Figure 2. - Definition of $x_{p}$ and $x_{y}$ 
The intermediate point and boundary constraints are specified at seven points, designated point 1 , point $2, \ldots$, point 7 , which divide the trajectory into six sub-arcs called First Stage, Second Stage A, Parking Orbit Coast, Second Stage B, TransLunar Coast, and Third Stage. All quantities with finite discontinuities will exhibit this by plus or minus superscripts on their subscripts; e.g., $\mathrm{m}_{2-}$ and $\mathrm{m}_{2+}$ indicate the mass at point 2 just prior to, and just after, separation of the first stage, respectively. The description on conditions specified on the six sub-arcs are as follows.

\section{First Stage}

Initially the vehicle is located at point 1, outside the atmosphere of the Earth with initial time, position vector, velocity vector, mass, thrust magnitude, and burning rate specified. Thus we have $t_{1}, \bar{x}_{1}, \bar{u}_{1}, m_{1}, F_{1}$, and $\beta_{1}$ given. This stage terminates at a given time $t_{2}$ when the empty fuel tank is separated. Therefore, the mass at the end of this arc is:

$$
m_{2-}=-\beta_{1}\left(t_{2}-t_{1}\right)+m_{1} \text {. }
$$

Throughout this arc, $F_{1}$ and $\beta_{1}$ are held fixed and the thrust direction is optimized.

\section{Second Stage A}

At point 2 , discontinuities in mass, thrust level, and burning rate occur. Thus we have:

$$
\begin{aligned}
& \mathrm{m}_{2+}=\mathrm{m}_{2-}+\Delta \mathrm{m}_{2} \\
& \mathrm{~F}_{2+}=\mathrm{F}_{1}+\Delta \mathrm{F}_{2} \\
& \mathrm{~B}_{2+}=B_{1}+\Delta B_{2}
\end{aligned}
$$

where $\Delta \mathrm{m}_{2}, \quad \Delta \mathrm{F}_{2}$, and $\Delta \beta_{2}$ are given. On this sub-arc the vehicle continues to thrust, varying its thrust direction angles optimally until it arrives in a parking orbit about the earth at point 3 . The parking orbit is partially specified by requiring: 


$$
\begin{aligned}
& \bar{x}_{3} \cdot \bar{x}_{3}=R^{2} \\
& \bar{u}_{3} \cdot \bar{u}_{3}=v^{2} \\
& \bar{u}_{3} \cdot \bar{x}_{3}=0
\end{aligned}
$$

where $R$ and $V$ are given quantities. This is equivalent to specifying the energy and magnitude of the angular momentum of the orbit and that the vehicle enter the orbit at an apsis. The plane of the orbit and the inclination of the orbit in the plane are left free to be optimized.

\section{Parking Orbit Coast}

When the orbit is achieved at point 3, the engines are switched off and discontinuities occur in thrust level and burning rate. Thus:

$$
\begin{aligned}
& F_{3}=F_{2+}-F_{2+}=0 \\
& B_{3}=B_{2+}-B_{2+}=0 .
\end{aligned}
$$

The vehicle coasts in this orbit until such time as it is best to resume thrusting again in order to enter a trans-lunar coasting arc. The mass is constant until point 4 is reached and coasting terminates.

\section{Second Stage B}

Engines are turned on again at point 4 and powered flight resumes. There are then discontinuities in $F$ and $B$ of magnitudes $F_{4+}$ and $\beta_{4+}$, respectively, and these quantities remain constant throughout this phase. The time $t_{4}$ is a quantity to be optimized. There is a restriction on the difference $t_{7}-t_{4}$ where $t_{7}$ is final time. This difference is the time of flight from the parking orbit to the lunar orbit and is a given number $T$. Thrusting continues under optimal steering until point 5 is reached at which point the trans-lunar coast begins. The time $t_{5}$ is also optimized. At this point the mass is given by

$$
m_{5-}=m_{2+}-\beta_{2+}\left(t_{3}-t_{2}\right)-\beta_{4+}\left(t_{5}-t_{4}\right) .
$$




\section{Trans-Lunar Coast}

At point 5 , the second stage is separated and coasting resumes. Thus:

$$
\begin{aligned}
& m_{5+}=m_{5-}+\Delta m_{5} \\
& F_{5}=F_{4+}-F_{4+}=0 \\
& \beta_{5}=B_{4+}-\beta_{4+}=0
\end{aligned}
$$

where $\Delta \mathrm{m}_{5}$ is specified. $\Delta \mathrm{m}_{5}$ can be considered the mass of the empty second stage. Any unburned fuel is treated as part of the payload. This phase takes the vehicle to the vicinity of the moon and terminates at point 6 , optimizing the time $t_{6}$.

\section{Third Stage}

At point 6, thrusting resumes under optimal steering. Thus, there are discontinuities in $F$ and $B$ of magnitudes $F_{6+}$ and $B_{6+}$, respectively, and these quantities are constant until the vehicle arrives in the specified lunar orbit. Point 7 is the terminal point at which five constraints describing the given lunar orbit must be satisfied. Thus:

$$
\psi_{i}\left(t_{7}, \bar{u}_{7}, \bar{x}_{7}\right)=0 \text { for } i=1,2, \ldots, 5 \text {. }
$$

These equations specify a conic section expressed relative to a rotating moon-centered coordinate system rigidly attached to the moon. These conditions are time-dependent because the coordinate system rotates with the moon. Recall that, as stated in Second Stage $\underline{B}$ above, the time $t_{7}$ was constrained to satisfy the equation

$$
\mathrm{t}_{7}-\mathrm{t}_{4}=\mathrm{T}
$$

where $T$ is a given number.

In summary, the purpose of this study, and the definition of optimal trajectory as used herein, is to determine the steering histories and switching times which yield a trajectory that is a mathematical extremum for placing maximum payload into a specified lunar orbit while satisfying the intermediate point and boundary constraints described above. 


\section{EULER-LAGRANGE EQUATIONS}

The work of Denbow ${ }^{1}$ has established that a necessary condition for an extremum of the generalized variational problem is that the classical Euler-Lagrange equations are satisfied on each sub-arc of a path subject to intermediate point constraints.

Note, that to be consistent with Ref. 1 , maximizing $\mathrm{m}_{7}$, the mass of the vehicle at point 7 , is equivalent to minimizing $-\mathrm{m}_{7}$.

Recalling the equations of motion in the previous section, the Euler-Lagrange sum is:

$$
\begin{aligned}
s & =\lambda_{1}\left(\dot{u}+\frac{F}{m} \sin x_{p} \cos x_{y}-x\right) \\
& +\lambda_{2}\left(\dot{v}-\frac{F}{m} \cos x_{p} \cos x_{y}-y\right) \\
& +\lambda_{3}\left(\dot{w}-\frac{F}{m} \sin x_{y}-z\right) \\
& +\lambda_{4}(\dot{x}-u) \\
& +\lambda_{5}(\dot{y}-v) \\
& +\lambda_{6}(\dot{z}-w) \\
& +\lambda_{7}(\dot{m}+B)
\end{aligned}
$$

The Euler-Lagrange equations can be determined from the conditions stated in the Introduction or equivalently from:

$$
\frac{d}{d t}\left(\frac{\partial s}{\partial \dot{\tau}}\right)=\frac{\partial s}{\partial \tau} .
$$

where $\tau=u, v, w, x, y, z, m, x_{p}$, and $x_{y}$. The resulting equations are: 


$$
\begin{aligned}
& \dot{\lambda}_{1}=-\lambda_{4} \\
& \dot{\lambda}_{2}=-\lambda_{5} \\
& \dot{\lambda}_{3}=-\lambda_{6} \\
& \dot{\lambda}_{4}=-\lambda_{1} \frac{\partial x}{\partial x}-\lambda_{2} \frac{\partial y}{\partial x}-\lambda_{3} \frac{\partial z}{\partial x} \\
& \dot{\lambda}_{5}=-\lambda_{1} \frac{\partial x}{\partial y}-\lambda_{2} \frac{\partial y}{\partial y}-\lambda_{3} \frac{\partial z}{\partial y} \\
& \dot{\lambda}_{6}=-\lambda_{1} \frac{\partial x}{\partial z}-\lambda_{2} \frac{\partial y}{\partial z}-\lambda_{3} \frac{\partial z}{\partial z} \\
& \dot{\lambda}_{7}=\frac{F}{m^{2}}\left(-\lambda_{1} \sin x_{p} \cos x_{y}+\lambda_{2} \cos x_{p} \cos x_{y}+\lambda_{3} \sin x_{y}\right) \\
& 0=\frac{F}{m}\left(\lambda_{1} \cos x_{p} \cos x_{y}+\lambda_{2} \sin x_{p} \cos x_{y}\right) \\
& 0=\frac{F}{m}\left(-\lambda_{1} \sin x_{p} \sin x_{y}+\lambda_{2} \cos x_{p} \sin x_{y}-\lambda_{3} \cos x_{y}\right)
\end{aligned}
$$

The last two equations yield:

$$
\begin{aligned}
& \tan x_{p}=-\frac{\lambda_{1}}{\lambda_{2}} \\
& \cos x_{p} \tan x_{y}=\frac{\lambda_{2} \lambda_{3}}{\lambda_{1}^{2}+\lambda_{2}^{2}} .
\end{aligned}
$$

The mathematical possibilities $\cos x_{y}=0$ and $F=0$ lead to trivial cases. The above equations can be written:

$$
\sin x_{p}=-\delta_{p} \frac{\lambda_{1}}{\sqrt{\lambda_{1}^{2}+\lambda_{2}^{2}}}
$$




$$
\begin{aligned}
& \cos x_{p}=\delta_{p} \frac{\lambda_{2}}{\sqrt{\lambda_{1}^{2}+\lambda_{2}^{2}}} \\
& \sin x_{y}=\delta_{y} \frac{\lambda_{3}}{\sqrt{\lambda_{1}^{2}+\lambda_{2}^{2}+\lambda_{3}^{2}}} \\
& \cos x_{y}=\delta_{y} \delta_{p} \frac{\sqrt{\lambda_{1}^{2}+\lambda_{2}^{2}}}{\sqrt{\lambda_{1}^{2}+\lambda_{2}^{2}+\lambda_{3}^{2}}}
\end{aligned}
$$

where $\delta_{p}= \pm 1$ and $\delta_{y}= \pm 1$. The ambiguities in the signs will be resolved in the next section. Note that we have the optimal steering angles, albeit in terms of the Lagrange multipliers.

If we define the quantity $\Lambda$ as:

$$
\Lambda=\sqrt{\lambda_{1}^{2}+\lambda_{2}^{2}+\lambda_{3}^{2}}
$$

and

$$
\bar{\lambda}=\left[\begin{array}{l}
\lambda_{I} \\
\lambda_{2} \\
\lambda_{3}
\end{array}\right], \bar{G}=\left[\begin{array}{l}
X \\
Y \\
Z
\end{array}\right],\left[\frac{\partial \bar{G}}{\partial \bar{x}}\right]=\left[\begin{array}{lll}
\frac{\partial X}{\partial X} & \frac{\partial Y}{\partial X} & \frac{\partial Z}{\partial X} \\
\frac{\partial X}{\partial Y} & \frac{\partial Y}{\partial Y} & \frac{\partial Z}{\partial Y} \\
\frac{\partial X}{\partial z} & \frac{\partial Y}{\partial z} & \frac{\partial Z}{\partial z}
\end{array}\right]
$$


- then the system of differential equations for the optimal trajectory can be summarized:

$$
\begin{aligned}
& \ddot{\ddot{x}}=\frac{F}{m} \delta_{y} \frac{\bar{\lambda}}{\bar{\Lambda}}+\bar{G} \\
& \dot{m}=-\beta \\
& \ddot{\bar{\lambda}}=\left[\frac{\partial \bar{G}}{\partial \bar{x}}\right] \bar{\lambda} \\
& \dot{\lambda}_{7}=\frac{F}{m^{2}} \delta_{y} \Lambda
\end{aligned}
$$

where the optimal steering is given by

$$
\begin{aligned}
& x_{p}=\tan ^{-1}\left(-\frac{\lambda_{1}}{\lambda_{2}}\right) \\
& x_{y}=\sin ^{-1}\left(\frac{\delta^{\lambda_{3}}}{\Lambda}\right) .
\end{aligned}
$$




\section{WEIERSTRASS NECESSARY CONDITION}

In Ref. 1, it is proved that the classical Weierstrass condition must hold on every sub-arc. Applied to this particular problem, this requirement reduces to specifying that the negative of the Euler-Lagrange sum, $\mathrm{S}$, is a maximum with respect to the control functions on the optimal path. Considering only the terms involving $x_{p}$ and $x_{y}$ and replacing these by their optimal values as found in the last section, the quantity to be maximized is:

$$
\delta_{Y} \frac{F}{m} \sqrt{\lambda_{1}^{2}+\lambda_{2}^{2}+\lambda_{3}^{2}}
$$

This constraint resolves the ambiguity in $\delta_{y} \cdot \delta_{y}$ is now seen to be +1 . Since $x_{y}$ has been restricted to the first and fourth quadrants, the equation for $\cos x_{y}$ in the preceding section indicates that $\delta_{y}$ and $\delta_{p}$ must have the same sign. Therefore, $\delta_{\mathrm{p}}=+1$. The optimal steering functions are now

$$
\begin{aligned}
& x_{p}=\tan ^{-1}\left(\frac{-\lambda_{1}}{\lambda_{2}}\right) \\
& x_{y}=\sin ^{-1}\left(\frac{\lambda_{3}}{\Lambda}\right)
\end{aligned}
$$

where the quadrant of $x_{p}$ and $x_{y}$ can be determined from the signs of $\lambda_{1}, \lambda_{2}$, and $\lambda_{3}$ in the usual manner with $-\frac{\pi}{2} \leq x_{y} \leq \frac{\pi}{2}$. 


\section{TRANSVERSALITY CONDITIONS}

Since the specified point constraints are insufficient to determine all required boundary conditions for the sub-arcs of the extremum, it is necessary to employ transversality conditions. We will apply the transversality relations of Denbow' ${ }^{1}$ generalized problem of Bolza to this particular problem. Each of these conditions applies to an end point of a sub-arc.

It can be shown that there exists a matrix, called the augmented matrix $\mathrm{A}^{\dagger}{ }^{\dagger}$ such that placing the proper restrictions on its rank yields all the necessary transversality conditions. The matrix $A$ for this problem is a $(20 \times 56)$ rectangular array with rank less than 20. Thus all (20 x 20) submatrices are singular. The term, $a_{i j}, i \neq 19,20$ of $A$ is a partial derivative of a point constraint with respect to a state variable. This matrix is shown in Figure 3 . If we designate by $c_{i}$ the quantities constrained at the end points of the sub-arcs, i. e., at point 1 , point $2, \ldots$ point 7, then there are 18 such terms and as $i$ takes on the integer values from 1 to $18, c_{i}$ takes on the values $t_{1}, u_{1}, v_{1}, w_{1}, x_{1}, y_{1}, z_{1}, m_{1}, t_{2}, \bar{u}_{3} \cdot \bar{u}_{3}, \bar{x}_{3} \cdot \bar{x}_{3}, \bar{u}_{3} \cdot \bar{x}_{3}$, $t_{7}-t_{4}, \psi_{1}, \psi_{2}, \psi_{3}, \psi_{4}, \psi_{5}$. Let $s_{j}$ designate an element of the time or state at each of the critical points, i.e., $s_{1}=t_{1}, s_{2}=u_{1}, s_{3}=v_{1}, s_{4}=w_{1}, s_{5}=x_{1}$, $\mathrm{s}_{6}=\mathrm{y}_{1}, \mathrm{~s}_{7}=\mathrm{z}_{1}, \mathrm{~s}_{8}=\mathrm{m}_{1}, \mathrm{~s}_{9}=\mathrm{t}_{2}, \mathrm{~s}_{10}=\mathrm{u}_{2}, \ldots \mathrm{s}_{55}=\mathrm{z}_{7}, \mathrm{~s}_{56}=\mathrm{m}_{7}$. There are 8 terms at each point and since there are 7 points, there are 56 values for $s_{j}$. The general term in the matrix can then be defined:

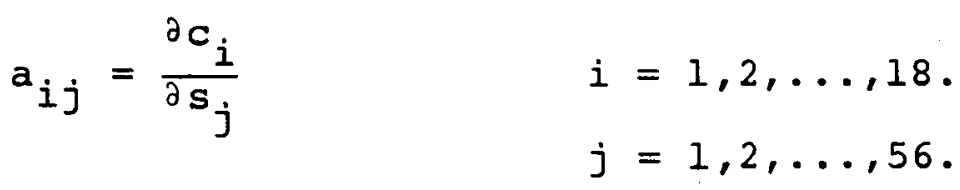

The 19th row is associated with the payoff quantity, maximum terminal mass, and contains -1 in the last column and zeros elsewhere. The 20th row consists of changes in the Hamiltonian and in the Lagrange multipliers evaluated at the seven endpoints of the sub-arcs. It is useful to divide the seven Lagrange multipliers into three parts and call them:

T The work was done in 1963 by R. W. Hunt, Southern Illinois University, in an unpublished report entitled "A Generalized Bolza-Mayer Problem with Discontinuous Solutions and Variable Intermediate Points, "for the Astrodynamic and Guidance Theory Division of the Aero-Astrodynamics Laboratory, George C. Marshall Space Flight Center, Huntsville, Ala. The paper by Denbow cited above gives sufficient information to construct the required matrix. 


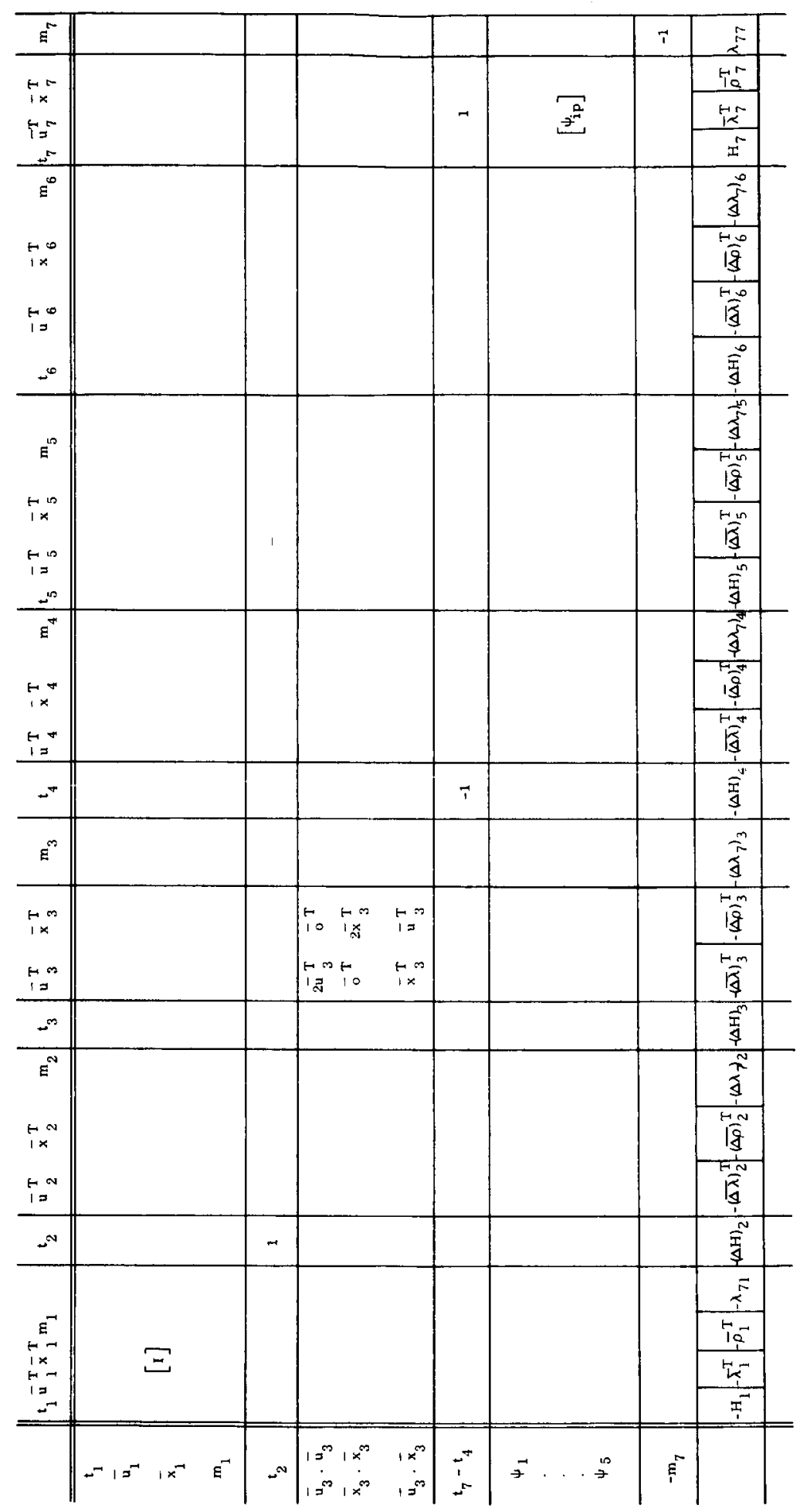

吾 


$$
\begin{aligned}
& \bar{\lambda}^{T}=\left(\lambda_{1}, \lambda_{2}, \lambda_{3}\right) \\
& -\rho^{T}=\left(\rho_{1}, \rho_{2}, \rho_{3}\right)=\left(\lambda_{4}, \lambda_{5}, \lambda_{6}\right) \\
& \lambda_{7}
\end{aligned}
$$

associating $\bar{\lambda}$ with the vector $\overline{\mathrm{u}}, \bar{\rho}$ with $\overline{\mathrm{x}}$ and $\lambda_{7}$ with mass. The Hamiltonian is given by

$$
\mathrm{H}=-\bar{\lambda} \cdot \dot{\overline{\mathrm{u}}}-\overline{\mathrm{p}} \cdot \dot{\overline{\mathrm{x}}}+\lambda_{7} \beta \cdot
$$

The 56 quantities in the 20th row are grouped into sets of eight, each set evaluated at one of the seven critical points. Thus the first eight elements are $-H_{T},-\lambda^{-T},-\rho^{-T},-\lambda 7$ each evaluated at point 1 , the next eight elements are $-\Delta H,-(\Delta \bar{\lambda})^{T},-(\Delta \bar{\rho})^{T}$ and $-\Delta \lambda_{7}$ evaluated at point 2 and similarly for points $3,4,5$, and 6 . The last eight elements are $H, \bar{\lambda}^{T}, \bar{\rho}^{T}, \lambda_{7}$ evaluated at point 7 .

We now impose upon $\mathrm{A}$ the condition that its rank be less than 20. By setting the determinants of all $20 \times 20$ submatrices of $\mathrm{A}$ to zero, we will have all the necessary transversality conditions. Many of the se determinants will be trivially zero and provide us with no information.

A careful study of the augmented matrix A reveals that many of the submatrices belong to a special form that makes obtaining some of the transversality conditions a matter of inspection. Consider the $(3 \times 6)$ submatrix associated with the parking orbit constraints,

$$
c_{3} \equiv\left[\begin{array}{rrrrrr}
2 u_{3} & 2 v_{3} & 2 w_{3} & 0 & 0 & 0 \\
0 & 0 & 0 & 2 x_{3} & 2 y_{3} & 2 z_{3} \\
x_{3} & y_{3} & z_{3} & u_{3} & v_{3} & w_{3}
\end{array}\right]
$$

This matrix must have rank 3 for these three constraints to be independent. Let $\mathrm{C}_{3}{ }^{*}$ be $(3 \times 3)$ non-singular sub-matrix of $\mathrm{C}_{3}$. In the same way, let the $(5 \times 7)$ submatrix $\mathrm{C}_{7}$ be that one associated with the terminal lunar orbit at point 7 . These represent five independent constraints and so must have rank 5 . Let $\mathrm{C}_{7}^{*}$ be any 
( $5 \times 5$ ) non-singular submatrix of $C_{7}$. We can now write a very convenient ( $19 \times 19$ ) submatrix of A using all rows except the last one. Thus:

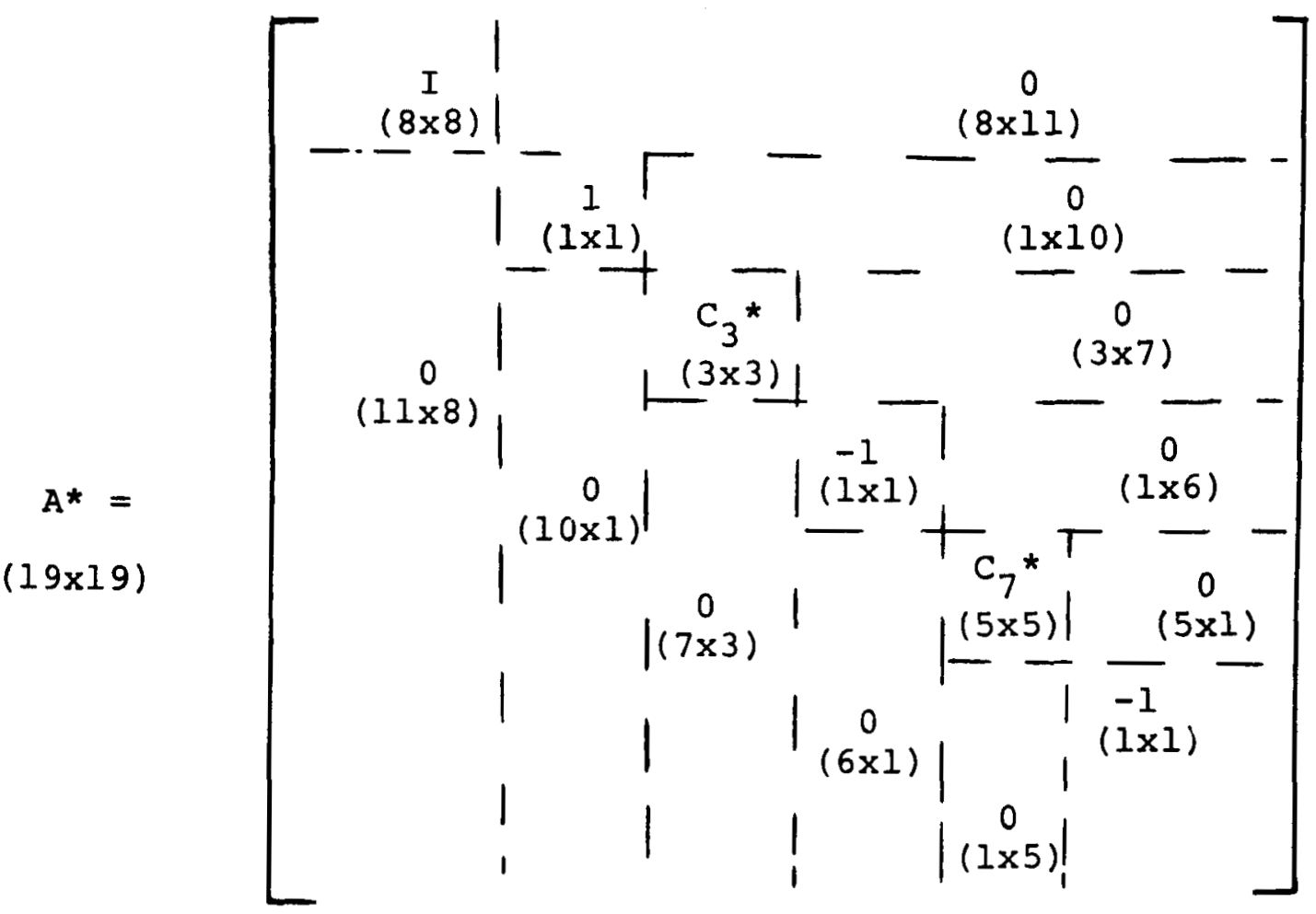

Note that to obtain the $(1 \times 6) 0$ matrix, the 25 th column was added to the 49 th column, an operation which does not change rank.

Since all $(20 \times 20)$ submatrices of A are singular, we can augment $A^{*}$ with any $(20 \times 1)$ column not already partly used in $A^{*}$ and the corresponding part of the last row, obtaining a singular $(20 \times 20)$ matrix. In particular, if this $(20 \times 1)$ column contains zeros in the first 19 rows, the last element in that column must vanish since $A^{*}$ is non-singular. There are 32 such columns, and thus we can obtain, by inspection, 32 transversality conditions:

$$
\begin{aligned}
& (\Delta \bar{\lambda})_{i}=0,(\Delta \bar{\rho})_{i}=0,\left(\Delta \lambda_{7}\right)_{i}=0 \text { for } i=2,4,5,6 . \\
& (\Delta H)_{i}=0 \text { for } i=3,5,6 \\
& \left(\Delta \lambda_{7}\right)_{3}=0
\end{aligned}
$$


This means that all the Lagrange multipliers are continuous at points $2,4,5$, and $6 ; \mathrm{H}$ is continuous at points 3,5 , and 6 ; and $\lambda_{7}$ is continuous all along the trajectory.

Consider now the $(16 \times 16)$ sub-matrix:

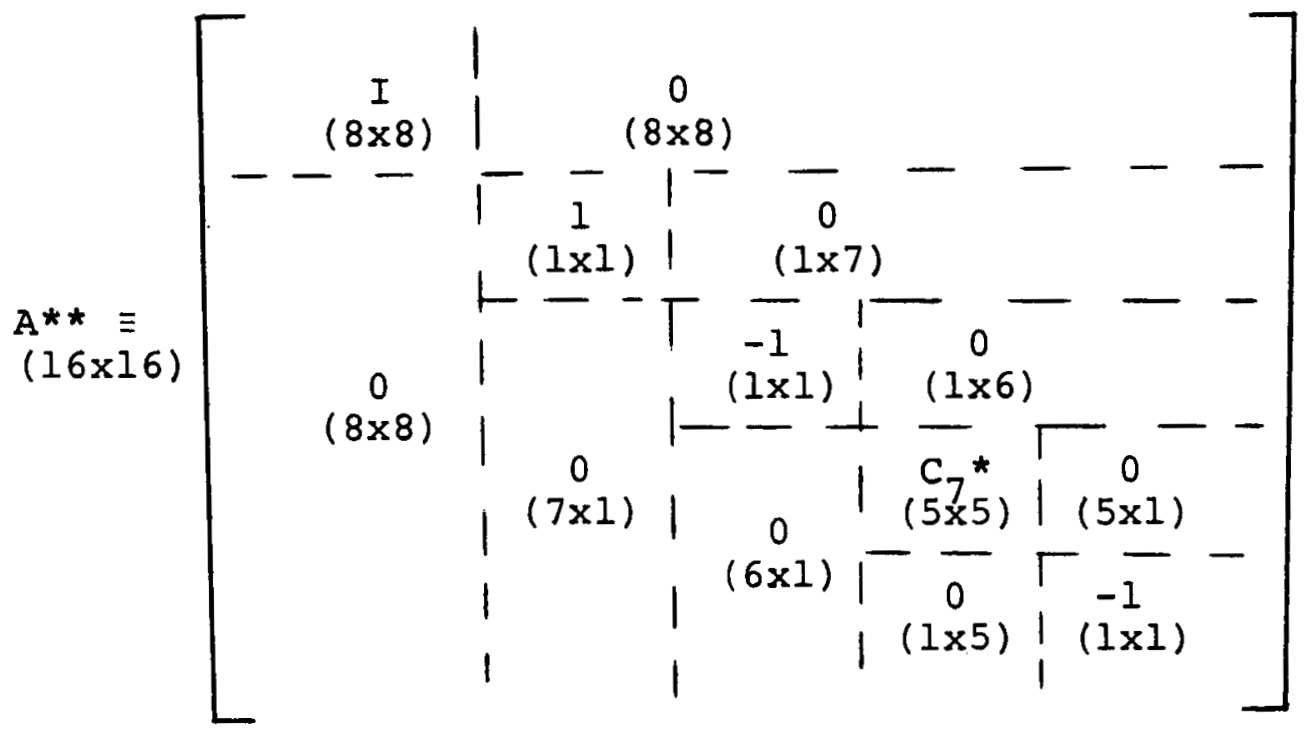

We need to form all possible $(20 \times 20)$ matrices using $\mathrm{A}^{* *}$, contributions from the $(3 \times 6)$ matrix of parking orbit constraints $C_{3}$ and the last row. Since $A^{* *}$ is nonsingular, the $(4 \times 6)$ matrix:

$\left[\begin{array}{cccccc}2 u_{3} & 2 v_{3} & 2 w_{3} & 0 & 0 & 0 \\ 0 & 0 & 0 & 2 x_{3} & 2 y_{3} & 2 z_{3} \\ x_{3} & y_{3} & z_{3} & u_{3} & v_{3} & w_{3} \\ \left(-\Delta \lambda_{1}\right)_{3} & \left(-\Delta \lambda_{2}\right)_{3} & \left(-\Delta \lambda_{3}\right)_{3} & \left(-\Delta \rho_{1}\right)_{3} & \left(-\Delta \rho_{2}\right) & \left(-\Delta \rho_{3}\right)_{3}\end{array}\right]$

has rank less than 4 . Thus, all $(4 \times 4)$ determinants vanish. This constraint yields three transversality conditions. We can write the above $(4 \times 6)$ matrix in a more concise manner as: 


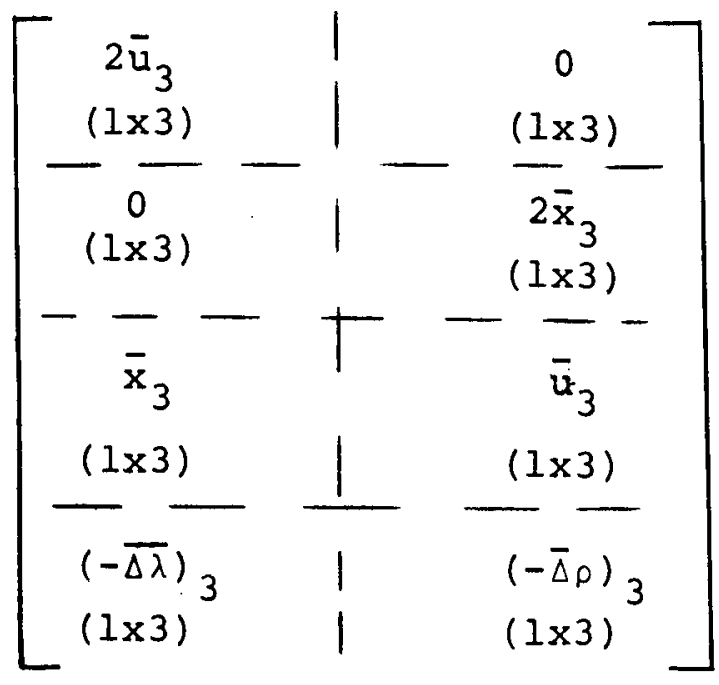

If we post-multiply this matrix by the following $(6 \times 6)$ matrix, rank will be conserved.

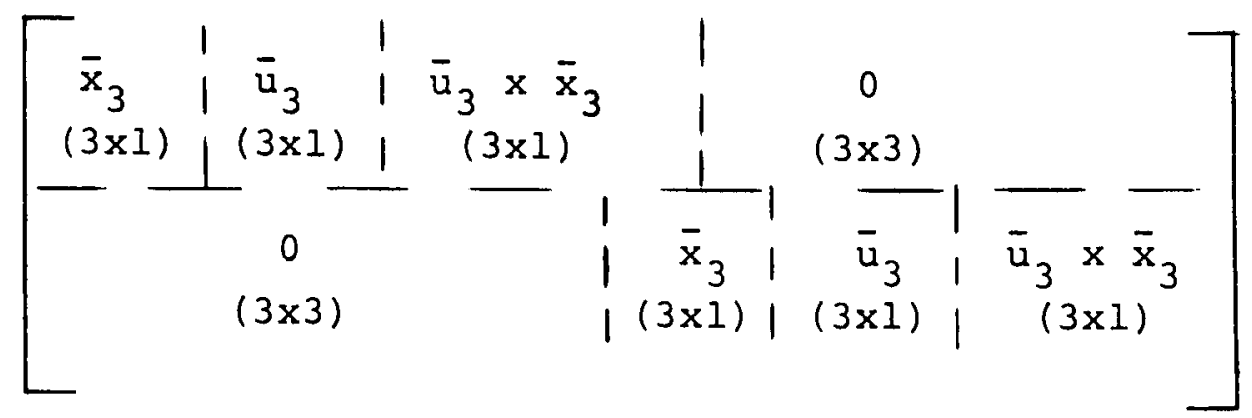

The product is a ( $4 \times 6)$ matrix of rank less than 4 and, using the relations describing the parking orbit constraints, this matrix is:

$\left[\begin{array}{cccccc}0 & 2 \mathrm{v}^{2} & 0 & 0 & 0 & 0 \\ 0 & 0 & 0 & 2 \mathrm{R}^{2} & 0 & 0 \\ \mathrm{R}^{2} & 0 & 0 & 0 & \mathrm{v}^{2} & 0 \\ (-\overline{\Delta \lambda})_{3} \cdot \overline{\mathrm{x}}_{3} & (-\overline{\Delta \lambda})_{3} \cdot \overline{\mathrm{u}}_{3} & (-\overline{\Delta \lambda})_{3} \cdot\left(\overline{\mathrm{u}}_{3} \times \overline{\mathrm{x}}_{3}\right) & (-\overline{\Delta \rho})_{3} \cdot \overline{\mathrm{x}}_{3} & (-\overline{\Delta \rho})_{3} \cdot \overline{\mathrm{u}}_{3} & (-\overline{\Delta \rho})_{3} \cdot\left(\overline{\mathrm{u}}_{3} \times \overline{\mathrm{x}}_{3}\right)\end{array}\right]$

The vanishing of all $(4 \times 4)$ determinants yields the transversality conditions: 


$$
\begin{aligned}
& (\overline{\Delta \lambda})_{3} \cdot\left(\bar{u}_{3} \times \bar{x}_{3}\right)=0 \\
& (\overline{\Delta p})_{3} \cdot\left(\bar{u}_{3} \times \bar{x}_{3}\right)=0 \\
& \mathrm{R}^{2}(\overline{\Delta p})_{3} \cdot \bar{u}_{3}=v^{2}(\overline{\Delta \lambda})_{3} \cdot \bar{x}_{3}
\end{aligned}
$$

The first two equations imply that the vectors $(\overline{\Delta \lambda})_{3}$ and $(\overline{\Delta \rho})_{3}$ are in the plane of $\overline{\mathrm{u}}_{3}$ and $\overline{\mathrm{x}}_{3}$, i.e., the flight plane. Note also that the last matrix above has rank 3 which confirms the requirement that our three parking orbit constraints are independent.

The last two transversality conditions can be obtained by considering the (14 $\times 14)$ sub-matrix:

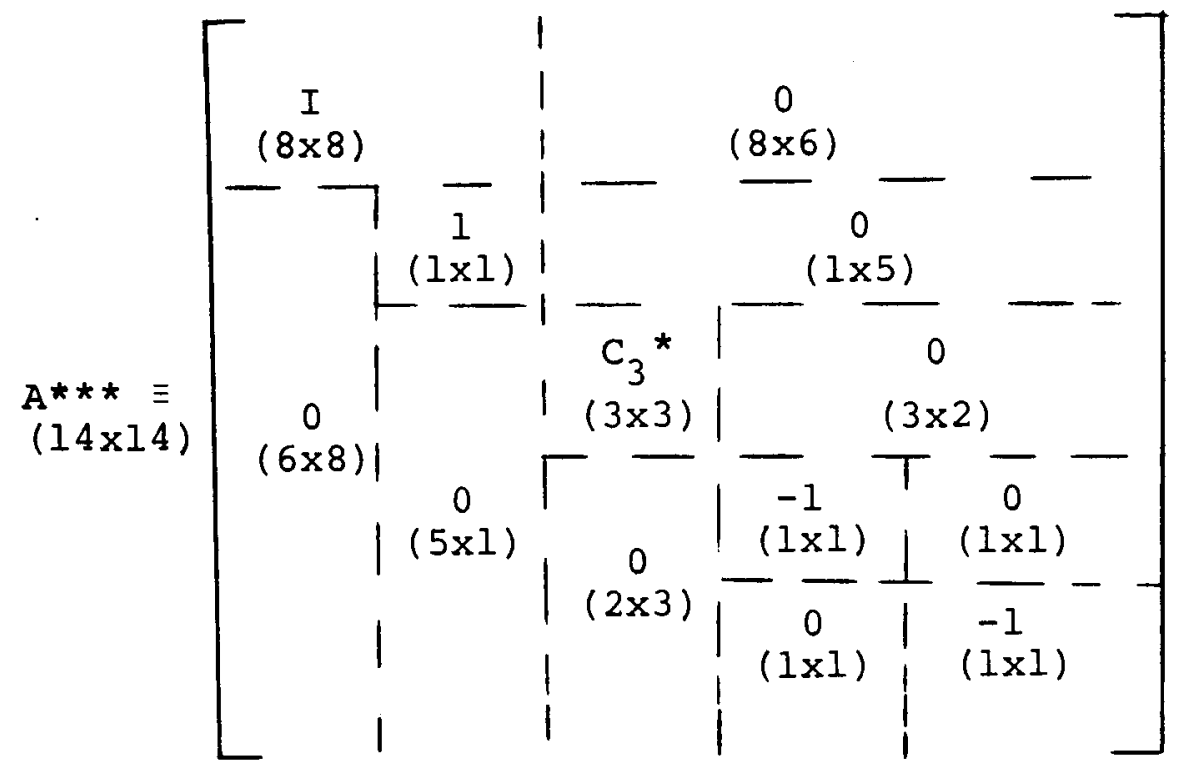

We need now to join this matrix to a $(6 \times 6)$ one formed by considering the $(5 \times 7)$ submatrix associated with the terminal constraints and the contributions from the last row. Since $A^{* * *}$ is non-singular, the $(6 \times 7)$ matrix, $M$ 


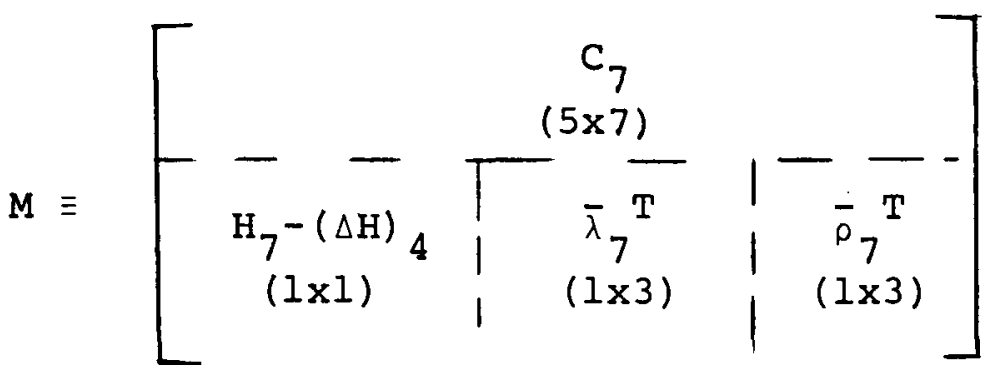

has rank less than 6. The term $\mathrm{H}_{7}-\Delta \mathrm{H}_{4}$ arises from the need to eliminate the 1 in the 49 th column by adding to the 49 th column the 25 th column. Such column operations leave rank invariant. The two remaining transversality conditions are obtained from this $(6 \times 7)$ matrix. We need now to impose upon the system the desired terminal constraint that the vehicle be in a specified lunar orbit. The lunar orbit is defined in a moon-centered coordinate system and so it is convenient at this point to digress for a description of the system and the establishment of a few useful relations.

We recall that the vector equation:

$$
\bar{\psi}\left(x_{s}, y_{s}, z_{s}, u_{s}, v_{s}, w_{s}\right) \equiv\left[\psi_{1}, \psi_{2}, \ldots, \psi_{5}\right] T=0
$$

describes a lunar orbit which is a conic section with respect to a coordinate system rigidly attached to the moon and having a focus at the center of the moon. In this system, the position and velocity vectors of the vehicle are:

$$
\begin{aligned}
& \bar{x}_{s} \equiv\left[x_{s}, y_{s}, z_{s}\right]^{T} \\
& \bar{u}_{s} \equiv\left[u_{s}, v_{s}, w_{s}\right]^{T}
\end{aligned}
$$

The coordinate system is chosen in such a manner that the conic lies in the $x_{s} y_{S}$ plane, the major axis is co-linear with the $x_{S}$ axis, and the directrix associated with the focus at the moon crosses the positive $x_{S}$ axis.

Let $\mathrm{C}$ be the $(3 \times 3)$ matrix such that the position of the vehicle with respect to the moon-centered system at time $t_{7}$, the terminal time, is $\bar{x}_{s}=c\left(\bar{x}_{7}-\bar{x}_{q}\right)$ where $\bar{x}_{d}$ is the position of the moon with respect to the Earth-centered reference frame at time $t_{7}$. Then: 


$$
\bar{u}_{s}=c\left(\bar{u}_{7}-\dot{\bar{x}}_{6}\right)+\dot{c}\left(\bar{x}_{7}-\bar{x}_{6}\right)
$$

and

$$
r_{s}=\sqrt{\left(x_{7}-x_{l}\right)^{2}+\left(y_{7}-y_{l}\right)^{2}+\left(z-z_{l}\right)^{2}}
$$

Let

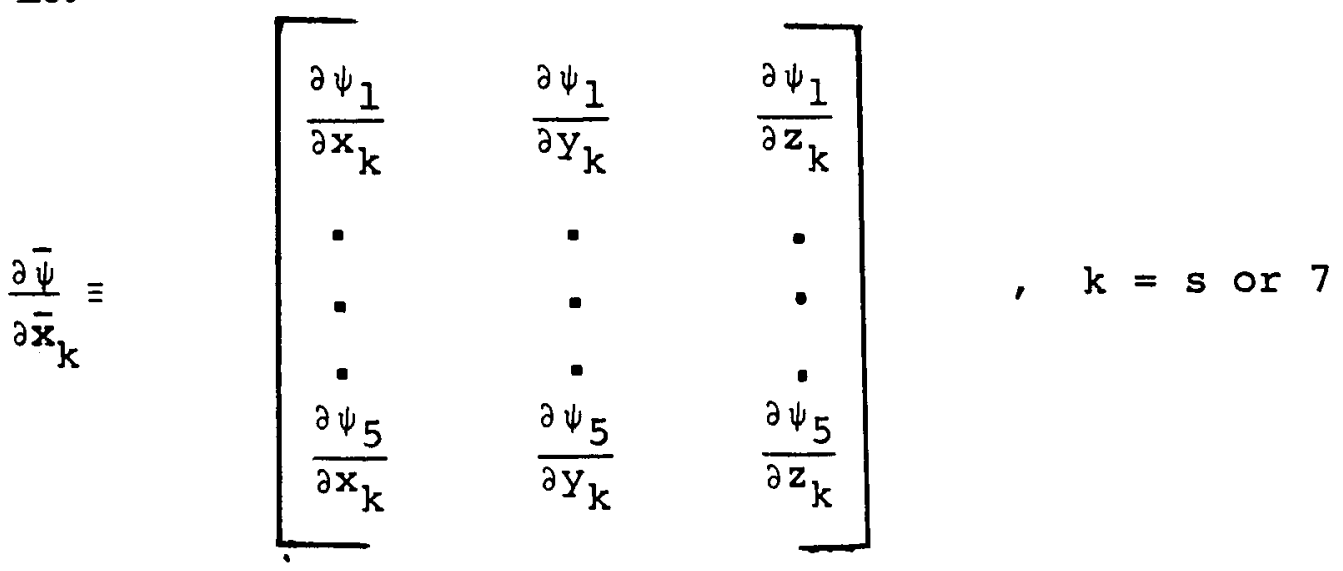

be a $(5 \times 3)$ matrix. Similarly for $\frac{\partial \bar{\psi}}{\partial \bar{u}_{k}}$.

Also:

$\frac{\partial \bar{x}_{s}}{\partial \bar{x}_{7}} \equiv\left[\begin{array}{ccc}\frac{\partial x_{s}}{\partial x_{7}} & \frac{\partial x_{s}}{\partial y_{7}} & \frac{\partial x_{s}}{\partial z_{7}} \\ \frac{\partial y_{s}}{\partial x_{7}} & \frac{\partial y_{s}}{\partial y_{7}} & \frac{\partial y_{s}}{\partial z_{7}} \\ \frac{\partial z_{s}}{\partial z_{7}} & \frac{\partial z_{s}}{\partial y_{7}} & \frac{\partial z_{s}}{\partial z_{7}}\end{array}\right]$

Similarly for $\frac{\partial \bar{u}_{s}}{\partial \bar{u}_{7}}$ and $\frac{\partial \bar{u}_{s}}{\partial \bar{x}_{7}}$, 
and

$$
\frac{\partial \bar{x}_{k}}{\partial t} \equiv\left[\frac{\partial x_{k}}{\partial t}, \frac{\partial y_{k}}{\partial t}, \frac{\partial z_{k}}{\partial t}\right]^{T}, k=s, 7 ;
$$

similarly for $\frac{\partial \vec{u}_{k}}{\partial t}$.

From the above definitions, one can readily establish the following relations:

$$
\begin{aligned}
& \frac{\partial \bar{x}_{s}}{\partial \bar{x}_{7}}=C \\
& \frac{\partial \bar{u}_{s}}{\partial \bar{u}_{7}}=C \\
& \frac{\partial \bar{x}_{s}}{\partial \bar{u}_{7}}=0 \\
& \frac{\partial \bar{u}_{s}}{\partial \bar{x}_{7}}=\dot{C} \\
& \frac{\partial \bar{\psi}}{\partial t}=\frac{\partial \bar{\psi}}{\partial \bar{x}_{s}} \quad \frac{\partial \overline{\mathbf{x}}_{s}}{\partial t}+\frac{\partial \bar{\psi}}{\partial \bar{u}_{s}} \quad \frac{\partial \bar{u}_{s}}{\partial t} \\
& \frac{\partial \bar{\psi}}{\partial \bar{x}_{7}}=\frac{\partial \bar{\psi}_{\bar{\psi}}}{\partial \bar{x}_{s}} \quad \frac{\partial \bar{x}_{s}}{\partial \bar{x}_{7}}+\frac{\partial \bar{\psi}}{\partial \bar{u}_{s}} \frac{\partial \bar{u}_{s}}{\partial \bar{x}_{7}}=\frac{\partial \bar{\psi}}{\partial \bar{x}_{s}} c+\frac{\partial \bar{\psi}}{\partial \bar{u}_{s}} \dot{c} \\
& \frac{\partial \psi}{\partial \bar{u}_{7}}=\frac{\partial \bar{\psi}_{s}}{\partial \bar{x}_{s}} \frac{\partial \bar{x}_{s}}{\partial \bar{u}_{7}}+\frac{\partial \bar{\psi}_{s}}{\partial \bar{u}_{s}} \frac{\partial \bar{u}_{s}}{\partial \bar{u}_{7}}=0+\frac{\partial \bar{\psi}_{s}}{\partial \bar{u}_{s}} C
\end{aligned}
$$


Now, we return to the task of extracting two transversality conditions from the matrix $M$, in which $C_{7}$ is shown explicitly. $M$ is a $(6 \times 7)$ matrix which can be partitioned:

$$
\mathbf{M}=\left[\begin{array}{ccccc}
\frac{\partial \bar{\psi}}{\partial t} & \mid & \frac{\partial \bar{\psi}}{\partial \bar{u}_{7}} & \mid & \overline{\partial \bar{\psi}} \\
(5 \times 1) & \mid & (5 \times 3) & \mid & (5 \times 3) \\
- & + & - & - & - \\
\mathrm{H}_{7}-(\Delta \mathrm{H}) & 1 & \bar{\lambda}_{7}^{T} & \mid & \bar{\rho}_{7}^{T} \\
(1 \times 1) & \mid & (1 \times 3) & \mid & (1 \times 3)
\end{array}\right]
$$

This matrix has rank less than six. Thus all $(6 \times 6)$ submatrices are singular. The setting of two non-trivially vanishing determinants to zero yields the remaining necessary conditions. But the matrix $M$ is not in convenient form. It can, however, be transformed into a very simple form by a sequence of multiplications by elementary matrices. These operations conserve rank. Before we embark on this procedure, the matrix $M$ can be written in terms of the moon-centered coordinate system by using the relations stated above. Thus, we have:

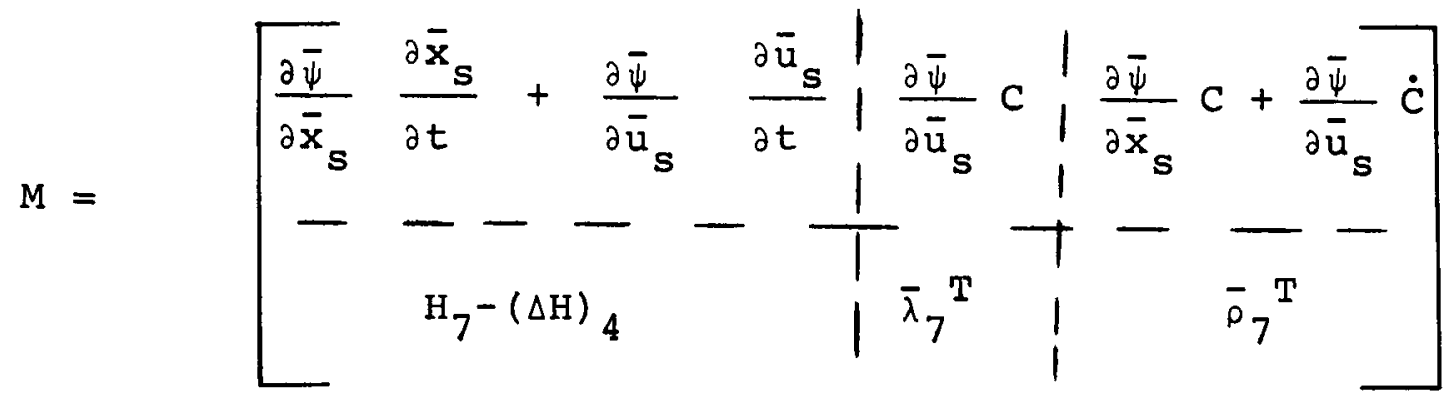

Careful examination of the partitioned matrices will reveal which multiplications by elementary matrices are useful for studying the rank of $M$. Notice that the two upper right hand submatrices contain the term $\underline{\partial \bar{\Psi}}$. This suggests that postmultiplication by the ( $7 \times 7$ ) non-singular matrix: $\partial \bar{u}_{s}$ 


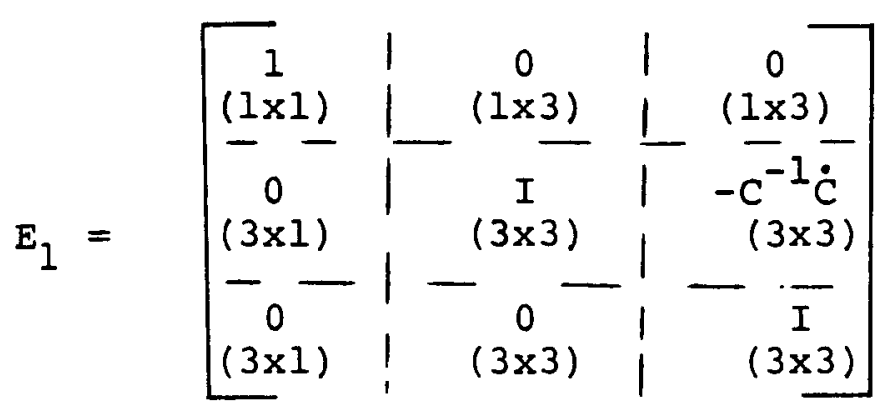

will eliminate the second term in the upper right submatrix. Thus we have:

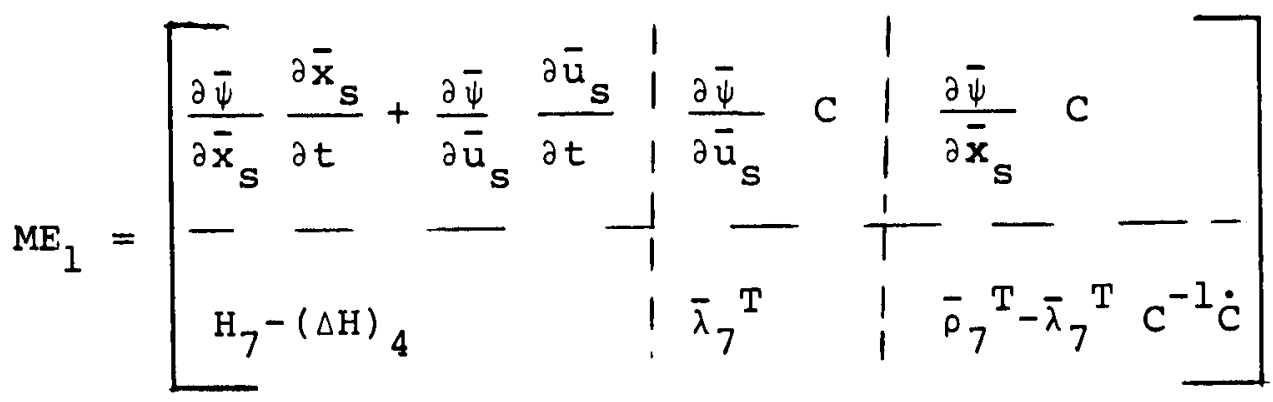

In a similar way, we can use the upper right submatrices to null the upper left submatrix. Consider the $(7 \times 7)$ non-singular matrix

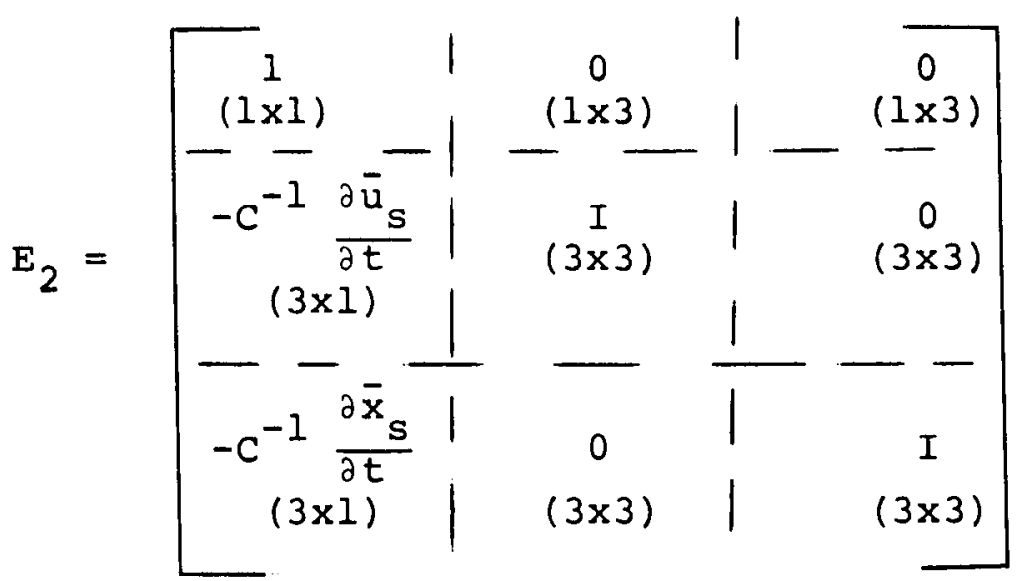


Post-multiplication yields:

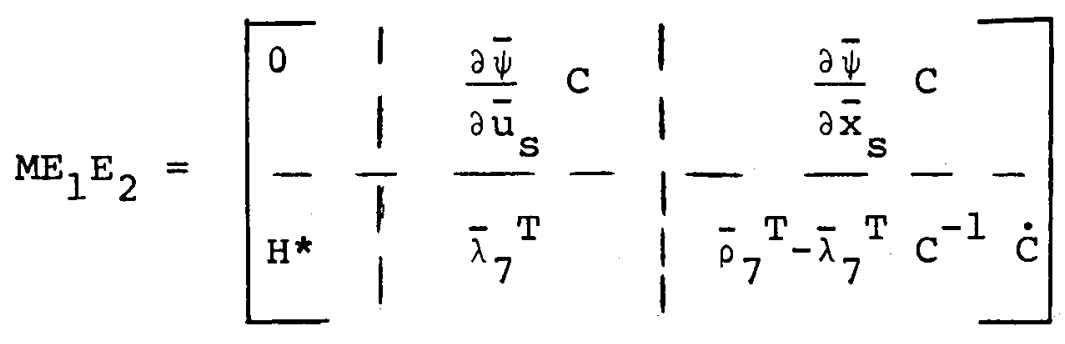

where

$$
\mathrm{H}^{*} \equiv \mathrm{H}_{7}-(\Delta \mathrm{H})_{4} \overline{\bar{\lambda}}_{7}^{\mathrm{T}} \mathrm{C}^{-1} \frac{\partial \overline{\mathrm{u}}_{\mathrm{S}}}{\partial t}-\left(\bar{\rho}_{7}^{\mathrm{T}}-\bar{\lambda}_{7}^{\mathrm{T}} \mathrm{C}^{-1} \dot{\mathrm{C}}\right) \mathrm{C}^{-1} \frac{\partial \overline{\mathrm{x}}_{\mathrm{s}}}{\partial t}
$$

To eliminate the terms $\mathrm{C}$, we use:

$$
\mathrm{E}_{3}=\left[\begin{array}{ccc}
1 & 0 & 0 \\
0 & \mathrm{C}^{-1} & 0 \\
0 & 0 & \mathrm{C}^{-1}
\end{array}\right]
$$

Thus:

$$
M * \equiv E_{1} E_{2} E_{3}=\left[\begin{array}{ccc}
0 & \frac{\partial \bar{\psi}}{\partial \bar{u}_{S}} & \frac{\partial \bar{\psi}}{\partial \bar{x}_{S}} \\
H^{*} & \lambda * & \rho *
\end{array}\right]
$$

where

$$
\begin{aligned}
& \bar{\lambda} *=\left(\lambda_{1} *, \lambda_{2} *, \lambda_{3} *\right)=\bar{\lambda}_{7}^{\mathrm{T}} \mathrm{C}^{-1} \\
& \bar{\rho} *=\left(\rho_{1} *, \rho_{2} *, \rho_{3}^{*}\right)=\left(\bar{\rho}_{7}^{\mathrm{T}}-\bar{\lambda}_{7}^{\mathrm{T}} \mathrm{C}^{-1} \dot{\mathrm{C}}\right) \mathrm{C}^{-1}
\end{aligned}
$$

29 
By the way, the inverse $\mathrm{C}^{-1}$ we have been using so liberally, does exist and, in fact, will be replaced by $\mathrm{C}^{\mathrm{T}}$ since $\mathrm{C}$ is orthogonal. Further simplifications require the specification of $\bar{\psi}$.

An arbitrary lunar orbit can be described in the following way. We choose our moon-centered coordinate system after the orbit is specified. We choose the $x_{s}, y_{s}$ plane to be the plane of the orbit and so $z_{s}=0$ and $r_{s}^{2}=x_{s}^{2}+y_{s}^{2}$. The constants are $e$, the eccentricity of the orbit, $\ell$, the semi-latus rectum and $\mu_{c}$, the gravitational constant of the moon, i.e., the product of the universal constant of gravitation and the mass of the moon. The following equations may be derived directly from Kepler's laws:

$$
\begin{aligned}
& \psi_{1} \equiv u_{s}+\frac{y_{s}}{r_{s}} \sqrt{\frac{\mu_{l}}{l}}=0 \\
& \psi_{2} \equiv v_{s}-\left(\frac{x_{s}}{r_{s}}+e\right) \sqrt{\frac{\psi_{l}}{l}}=0
\end{aligned}
$$

$$
\psi_{3} \equiv w_{s}=0
$$

$$
\psi_{4} \equiv r_{s}+e x_{s}-\ell=0
$$

$$
\psi_{5} \equiv z_{s}=0
$$

30 
Now, it is useful to display $M^{*}$ completely.

$M *=\left[\begin{array}{ccccccc}0 & 1 & 0 & 0 & \frac{\partial \psi_{1}}{\partial x_{s}} & \frac{\partial \psi_{1}}{\partial y_{s}} & 0 \\ 0 & 0 & 1 & 0 & \frac{\partial \psi_{2}}{\partial x_{s}} & \frac{\partial \psi_{2}}{\partial y_{s}} & 0 \\ 0 & 0 & 0 & 1 & 0 & 0 & 0 \\ 0 & 0 & 0 & 0 & \frac{\partial \psi_{4}}{\partial x_{s}} & \frac{\partial \psi_{4}}{\partial y_{s}} & 0 \\ 0 & 0 & 0 & 0 & 0 & 0 & 1 \\ H^{*} & \lambda_{1} * & \lambda_{2}^{*} & \lambda_{3} * & \rho_{1} * & \rho_{2}{ }^{*} & \rho_{3}^{*}\end{array}\right]$

The following non-singular matrix will be used to yield an even simpler matrix.

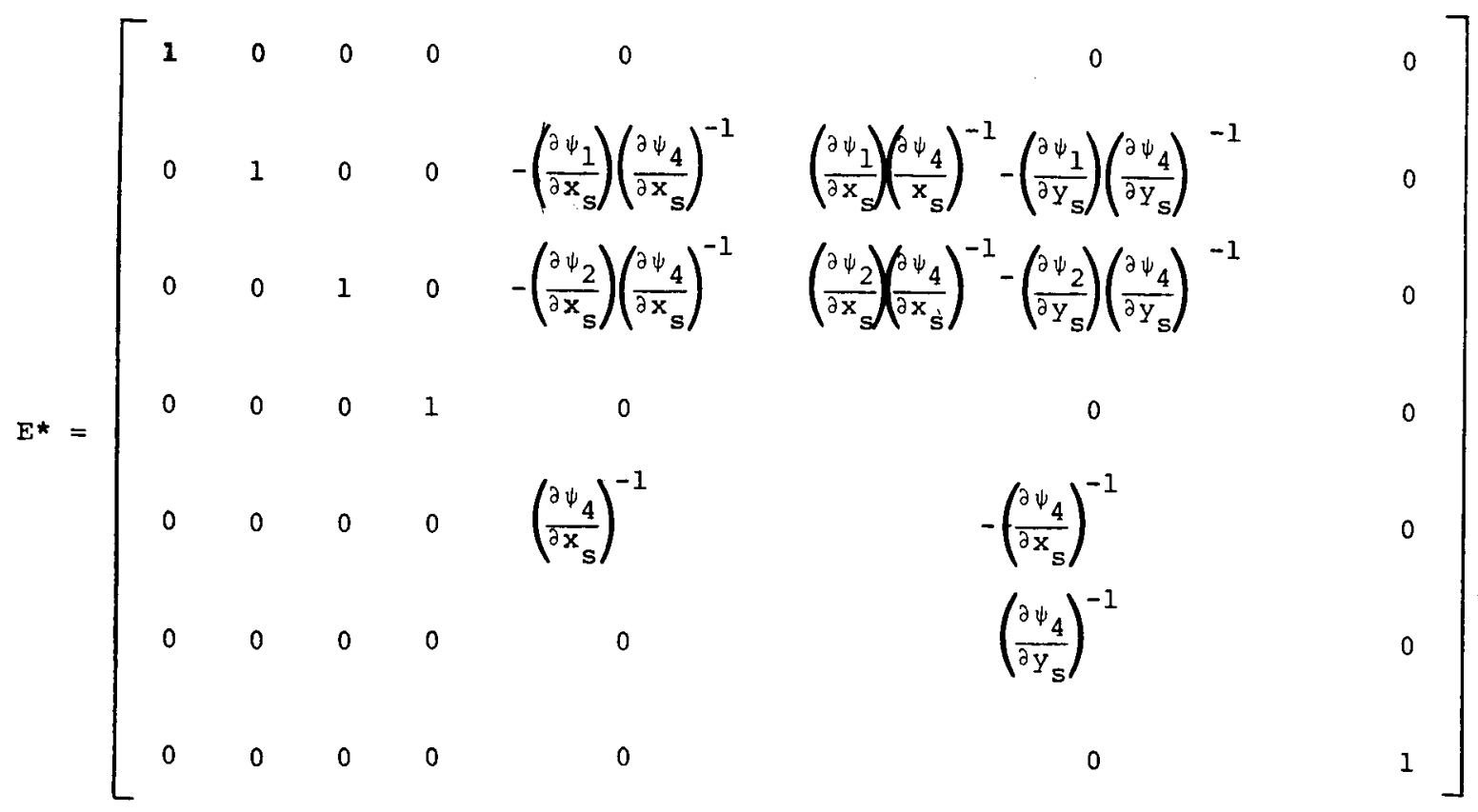


provided $\frac{\partial \psi_{4}}{\partial x_{S}} \neq 0$ and $\frac{\partial \psi_{4}}{\partial y_{S}} \neq 0$. Thus:

$\mathrm{M} * \mathrm{E}^{*}=\left[\begin{array}{ccccccc}0 & 1 & 0 & 0 & 0 & 0 & 0 \\ 0 & 0 & 1 & 0 & 0 & 0 & 0 \\ 0 & 0 & 0 & 1 & 0 & 0 & 0 \\ 0 & 0 & 0 & 0 & 1 & 0 & 0 \\ H^{*} & \lambda_{1} & \lambda_{2} & \lambda_{3}{ }^{*} & \rho_{1} * & \rho_{2} * & \rho_{3}\end{array}\right]$

where

$$
\begin{aligned}
\rho_{1} * * & -\lambda_{1} * \frac{\partial \psi_{1}}{\partial x_{s}}\left(\frac{\partial \psi_{4}}{\partial x_{s}}\right)^{-1}-\lambda_{2} * \frac{\partial \psi_{2}}{\partial x_{s}}\left(\frac{\partial \psi_{4}}{\partial x_{s}}\right)^{-1}+\rho_{1} *\left(\frac{\partial \psi_{4}}{\partial x_{s}}\right)^{-1} \\
\rho_{2} * * & \lambda_{1} *\left[\frac{\partial \psi_{1}}{\partial x_{s}}\left(\frac{\partial \psi_{4}}{\partial x_{s}}\right)^{-1}-\frac{\partial \psi_{1}}{\partial y_{s}}\left(\frac{\partial \psi_{4}}{\partial y_{s}}\right)^{-1}\right] \\
& +\lambda_{2} *\left[\frac{\partial \psi_{2}}{\partial x_{s}}\left(\frac{\partial \psi_{4}}{\partial x_{s}}\right)^{-1}-\frac{\partial \psi_{2}}{\partial y_{s}}\left(\frac{\partial \psi_{4}}{\partial y_{s}}\right)^{-1}\right] \\
& -\rho_{1} *\left(\frac{\partial \psi_{4}}{\partial x_{s}}\right)^{-1}+\rho_{2} *\left(\frac{\partial \psi_{4}}{\partial y_{s}}\right)^{-1}
\end{aligned}
$$

It can be concluded here that $\mathrm{H}^{*}=\rho_{2}^{* *}=0$ if $\mathrm{M}^{*} \mathrm{E}^{*}$ is to have rank less than six. The matrix $M^{*} E^{*}$ was developed from $M$ by a sequence of multiplications by non-singular triangular matrices. These operations leave rank invariant. Thus we may direct our study of rank to the $(6 \times 7)$ matrix $M^{*} E^{*}$. We know its rank is less than six. Therefore, all $(6 \times 6)$ matrices are singular. Thus, $\mathrm{H}^{*}=0$ and $\mathrm{p}_{2} * *=0$. 
But replacing $\mathrm{p}_{2}{ }^{* *}$ by its value from its definition, we have:

$$
\begin{gathered}
\lambda_{1} *\left[\frac{\partial \psi_{1}}{\partial x_{s}}\left(\frac{\partial \psi_{4}}{\partial x_{s}}\right)^{-1}-\frac{\partial \psi_{1}}{\partial y_{s}}\left(\frac{\partial \psi_{4}}{\partial y_{s}}\right)^{-1}\right] \\
+\lambda_{2} *\left[\frac{\partial \psi_{2}}{\partial x_{s}}\left(\frac{\partial \psi_{4}}{\partial x_{s}}\right)^{-1}-\frac{\partial \psi_{2}}{\partial y_{s}}\left(\frac{\partial \psi_{4}}{\partial y_{s}}\right)^{-1}\right] \\
-\rho_{1} *\left(\frac{\partial \psi_{4}}{\partial x_{s}}\right)^{-1}+\rho_{2} *\left(\frac{\partial \psi_{4}}{\partial y_{s}}\right)^{-1}=0
\end{gathered}
$$

Now, $\psi_{1}, \psi_{2}$, and $\psi_{4}$ (from the Kepler equations for the lunar orbit) are:

$$
\begin{aligned}
& \psi_{1}=u_{s}+\frac{y_{s}}{\sqrt{x_{s}^{2}+y_{s}^{2}}} \sqrt{\frac{\mu_{l}}{\ell}} \\
& \psi_{2}=v_{s}-\left(\frac{x_{s}}{\sqrt{x_{s}^{2}+y_{s}^{2}}}+e\right) \sqrt{\frac{\mu_{l}}{\ell}} \\
& \Psi_{4}=\sqrt{x_{s}^{2}+y_{s}^{2}+e s_{s}-\ell}
\end{aligned}
$$

The required partial derivatives are then:

$$
\frac{\partial \psi_{1}}{\partial x_{s}}=-\frac{x_{s} y_{s}}{r_{s}{ }^{3}} \sqrt{\frac{\mu_{c}}{\ell}}
$$




$$
\begin{aligned}
& \frac{\partial \psi_{1}}{\partial y_{s}}=\frac{x_{s}^{2}}{r_{s}^{3}} \sqrt{\frac{\mu_{l}}{\ell}} \\
& \frac{\partial \psi_{2}}{\partial x_{s}}=-\frac{y_{s}^{2}}{r_{s}^{3}} \sqrt{\frac{\mu_{c}}{\ell}} \\
& \frac{\partial \psi_{2}}{\partial y_{s}}=+\frac{x_{s} y_{s}}{r_{s}} \sqrt{\frac{\mu_{c}}{\ell}} \\
& \frac{\partial \psi_{4}}{\partial y_{s}}=\frac{x_{s}}{r_{s}}+e \neq 0 \\
& \frac{\partial \psi_{4}}{\partial y_{s}}=\frac{y_{s}}{r_{s}} \neq 0
\end{aligned}
$$

The above transversality condition, using these relations, is then:

$$
\begin{aligned}
& \lambda_{1} *\left[\begin{array}{lll}
\frac{x_{s}^{y_{s}}}{r_{s}^{4}} & \sqrt{\frac{\mu_{l}}{l}}-\frac{x_{s}^{2}}{r_{s}^{3}} \sqrt{\frac{\mu_{l}}{\ell}}\left(\frac{x_{s}}{r_{s}}+e\right)
\end{array}\right] \\
& +\lambda_{2} *\left[\begin{array}{ll}
\frac{y_{s}^{3}}{r_{s}^{4}} & -\frac{x_{s} y_{s}}{r_{s}^{3}} \sqrt{\frac{\mu_{l}}{\ell}}\left(\frac{x_{s}}{r_{s}}+e\right)
\end{array}\right] \\
& -\rho_{1} * \frac{y_{s}}{r_{s}}+\rho_{2} *\left(\frac{x_{s}}{r_{s}}+e\right)=0
\end{aligned}
$$


Simplifying, we have:

$-\sqrt{\frac{\mu_{l}}{\ell}} \frac{\left(r_{s}+e x_{s}\right)}{r_{s}^{3}}\left(\lambda_{1} * x_{s}+\lambda_{2} * y_{s}\right)+\frac{-p_{1} * y_{s}+\rho_{2}\left(_{s}+e r_{s}\right)}{r_{s}}=0$

But the equations $\psi_{1}=\psi_{2}=\psi_{4}=0$ are equivalent to:

$$
\begin{aligned}
& \left(\frac{x_{s}}{r_{s}}+e\right) \sqrt{\frac{\mu_{l}}{\ell}}=v_{s} \\
& \frac{y_{s}}{r_{s}} \sqrt{\frac{\mu_{l}}{\ell}}=-u_{s} \\
& r_{s}+e x_{s}=\ell
\end{aligned}
$$

Using these relations, we have

$$
-\mu_{l} \ell \frac{\lambda_{1} * x_{s}+\lambda_{2} * y_{s}}{r_{s}^{3}}+\frac{\ell}{\mu_{0}}\left(+\rho_{1} * u_{s}+\rho_{2} * v_{s}\right)=0
$$

The definitions of $\bar{\lambda} *$ and $\bar{\rho} *$ are:

$$
\begin{aligned}
& \bar{\lambda} *=\left(\lambda_{1} *, \lambda_{2} *, \lambda_{3} *\right)=\bar{\lambda}_{7}^{T} C^{-1} \\
& \bar{\rho} *=\left(\rho_{1} *, \rho_{2} *, \rho_{3} *\right)=\left(\bar{\rho}_{7}{ }^{T}-\bar{\lambda}_{7} C^{-1} \dot{C}\right) c^{-1}
\end{aligned}
$$


and using the fact that $\mathrm{C}$ is an orthogonal matrix, we have:

$$
\frac{-\mu_{c}}{r_{s}{ }^{3}} \bar{\lambda}_{7}{ }^{T} C^{T} \bar{x}_{s}+\left(\bar{\rho}_{7}{ }^{T}-\bar{\lambda}_{7}{ }^{T} C^{T} \dot{C}\right) c^{T} u_{s}=0
$$

These last two transversality conditions are then:

$$
\begin{aligned}
& H_{7}-\left(\Delta_{4}\right)-\bar{\lambda}_{7} T_{C}^{T} \frac{\partial \bar{u}_{s}}{\partial t}-\left(\bar{p}_{7}{ }^{T}-\bar{\lambda}_{7}{ }^{T} C^{T} \dot{C}\right) c^{T} \frac{\partial \bar{x}_{s}}{\partial t}=0 \\
& -\frac{\mu_{Q}}{r_{s}{ }^{3}} \bar{\lambda}_{7}{ }^{T} C^{T} \bar{x}_{s}+\left(\bar{\rho}_{7}{ }^{T}-\bar{\lambda}_{7}{ }^{T} C^{T} \dot{C}\right) c^{T} \bar{u}_{s}=0
\end{aligned}
$$

36 


\section{THE SWITCHING FUNCTION}

The transversality conditions implying that the Hamiltonian is continuous at points 5 and 6 will now be examined closely. Thus, at point 5 , we have:

$$
\mathrm{H}_{5-}=\mathrm{H}_{5+}
$$

or equivalently,

$$
\begin{aligned}
& \bar{\lambda}_{5-} \cdot \dot{\bar{u}}_{5-}+\bar{\rho}_{5-} \cdot \dot{\bar{x}}_{5-}-\left(\lambda_{7}\right)_{5-} \beta_{5-}= \\
& \bar{\lambda}_{5+} \cdot \dot{\bar{u}}_{5+}+\bar{\rho}_{5+} \cdot \dot{\bar{x}}_{5+}-\left(\lambda_{7}\right)_{5+} \beta_{5 t}
\end{aligned}
$$

But other transversality conditions have shown that all the Lagrange multipliers are continuous at point 5 . Since $\bar{x}_{5}$ is also continuous and $B_{5+}$ is zero, we have:

$$
\left.\bar{\lambda}_{5} \cdot \dot{\bar{u}}_{5-}-\dot{\bar{u}}_{5+}\right)-\left(\lambda_{7}\right)_{5} \beta_{5-}=0
$$

Note that $\frac{\dot{u}}{\mathrm{u}}$ is acceleration and specific impulse is given by

$$
I_{s p}=\frac{F}{g_{o} \beta} \text {. }
$$

It was shown in Section $\mathrm{V}$ that:

$$
\frac{\ddot{x}}{\mathrm{x}}=\frac{\mathrm{F}}{\mathrm{m}} \quad \frac{\bar{\lambda}}{\bar{\Lambda}}+\mathrm{x}
$$

where $\Lambda=|\bar{\lambda}|$. 
Then the continuity of the Hamiltonian at point 5 implies:

$$
\frac{g_{0} I_{s p}}{m_{5-}} \Lambda_{5}-\left(\lambda_{7}\right)_{5}=0
$$

Similarly, the continuity of the Hamiltonian at point 6 implies:

$$
\frac{g_{0} I_{s p}}{m_{6+}} \Lambda_{6}-\left(\lambda_{7}\right)_{6}=0 .
$$

These conditions can be used as switching functions; points 5 and $6 \mathrm{can}$ be determined by the vanishing of these expressions.

If the switching function changes sign more than twice between points 4 and 7 , then one or more thrusting periods, intermediate to points 5 and 6 , may be required. 


\section{SUMMARY OF BOUNDARY CONDITIONS}

The conditions, which apply at the end points of the sub-arcs of an optimum trajectory and which cannot be trivially satisfied, are summarized below. In addition, the initial conditions which are unknown a priori at the endpoints of the sub-arcs are stated. A condition implying continuity of a quantity over a boundary point will not be stated and its value at the boundary point will not be considered to be free.

\section{Point 1}

Unspecified Quantities: $\lambda_{11}, \lambda_{31}, \bar{\rho}_{1}, \lambda_{71}\left(\lambda_{21}\right.$ is arbitrarily set equal to unity since all equations are homogenous in the Lagrange multipliers).

\section{Point 3}

Constraints: $\quad \bar{u}_{3} \cdot \bar{u}_{3}=v^{2}$

$$
\begin{aligned}
& \bar{x}_{3} \cdot \bar{x}_{3}=R^{2} \\
& \bar{u}_{3} \cdot \bar{x}_{3}=0
\end{aligned}
$$

$$
\begin{aligned}
& (\overline{\Delta \lambda})_{3} \cdot\left(\bar{u}_{3} \times \bar{x}_{3}\right)=0 \\
& (\overline{\Delta \rho})_{3} \cdot\left(\bar{u}_{3} \times \bar{x}_{3}\right)=0 \\
& v^{2}(\overline{\Delta \lambda})_{3} \cdot \bar{x}_{3}=R^{2}(\overline{\Delta \rho})_{3} \cdot \bar{u}_{3} \\
& (\Delta \mathrm{H})_{3}=0
\end{aligned}
$$

Unspecified Quantities: $\quad t_{3}, \bar{\lambda}_{3+}, \bar{\rho}_{3+}$ 


\section{Point 4}

Unspecified Quantity: $t_{4}$

\section{Point 7}

Constraints: $\quad \psi_{i}\left(t_{7}, \bar{u}_{7}, \bar{x}_{7}\right)=0 \quad(i=1,2,3,4,5)$

These last two transversality conditions are then:

$$
\mathrm{H}_{7},-\left(\Delta \mathrm{H}_{4}\right)-\bar{\lambda}_{7}{ }^{\mathrm{T}} \mathrm{C}^{\mathrm{T}} \frac{\partial \overline{\mathrm{u}}_{\mathrm{s}}}{\partial \mathrm{t}}-\left(\bar{\rho}_{7}{ }^{\mathrm{T}}-\bar{\lambda}_{7}{ }^{\mathrm{T}} \mathrm{C}^{\mathrm{T}} \dot{\mathrm{C}}\right) \mathrm{C}^{\mathrm{T}} \frac{\partial \overline{\mathrm{x}}_{\mathrm{s}}}{\partial \mathrm{t}}=0
$$

and

$$
-\frac{\mu}{r_{s}{ }^{3}} \bar{\lambda}_{7}{ }^{T} C^{T} \bar{x}_{s}+\left(\bar{\rho}_{7}{ }^{T}-\bar{\lambda}_{7}{ }^{T} C^{T} \dot{c}\right) C^{T} \bar{u}_{s}=0
$$

There are a total of 14 unknown quantities in the problem, precisely enough to satisfy the 14 conditions. Some of these conditions can be easily satisfied; for example, one can integrate the differential equations up until the time at which $\bar{x}_{3} \cdot \bar{x}_{3}=R^{2}$; i.e., $t_{3}$ can be chosen to be that time at which the distance from the Earth's center is R.

National Aeronautics and Space Administration

Electronics Research Center

Cambridge, Massachusetts, October 1966

129-04-04-07-25 
